\title{
Constrained Community-Based Gene Regulatory Network Inference
}

\author{
FERDINANDO FIORETTO, New Mexico State University \& University of Udine \\ AGOSTINO DOVIER, University of Udine \\ ENRICO PONTELLI, New Mexico State University
}

\begin{abstract}
The problem of gene regulatory network inference is a major concern of systems biology. In recent years, a novel methodology has gained momentum, called community network approach. Community networks integrate predictions from individual methods in a "metapredictor," in order to compose the advantages of different methods and soften individual limitations. This article proposes a novel methodology to integrate prediction ensembles using constraint programming, a declarative modeling and problem solving paradigm. Constraint programming naturally allows the modeling of dependencies among components of the problem as constraints, facilitating the integration and use of different forms of knowledge. The new paradigm, referred to as constrained community network, uses constraints to capture properties of the regulatory networks (e.g., topological properties) and to guide the integration of knowledge derived from different families of network predictions. The article experimentally shows the potential of this approach: The addition of biological constraints can offer significant improvements in prediction accuracy.
\end{abstract}

Categories and Subject Descriptors: J.3 [Life and Medical Sciences]: Biology and Genetics

General Terms: Algorithms, Experimentation

Additional Key Words and Phrases: Gene regulatory networks, bioinformatics, constraint programming

\section{ACM Reference Format:}

Ferdinando Fioretto, Agostino Dovier, and Enrico Pontelli. 2015. Constrained community-based gene regulatory network inference. ACM Trans. Model. Comput. Simul. 25, 2, Article 11 (February 2015), 26 pages. DOI: http://dx.doi.org/10.1145/2688909

\section{INTRODUCTION}

In a cellular context, genes interact to orchestrate a variety of fundamental tasks, such as the response of cells to environmental stimuli (e.g., a drug), the cell proliferation, and its apoptosis (i.e., cell death). Research in the field of systems biology has highlighted the importance of investigating such interactions at the different levels in which they occur, in order to provide a comprehensive understanding of the behavior of a biological system. At the cellular level, proteins are considered to be amongst the most important components to carry out those functions which are necessary for cell regulation. In simple terms, the information needed to produce proteins is encoded within the genes. The process used to express a protein can be abstracted as a two-step process: First, the information of coding DNA (gene) is transcribed into a messenger RNA (mRNA)

The research has been supported by NSF grants HRD-1345232, CNS-1042341, DGE-0947465, and GNCS 2014.

Authors' addresses: Ferdinando Fioretto and Enrico Pontelli, Dept. Computer Science, New Mexico State University, Box 30001, MSC CS, Las Cruces, NM 88003; emails: \{ffiorett, epontell\}@cs.nmsu.edu; Agostino Dovier, Dip. Matematica e Informatica, Università degli Studi di Udine, Via delle Scienze, 208, Udine 33100, Italy; email: agostino.dovier@uniud.it.

Permission to make digital or hard copies of part or all of this work for personal or classroom use is granted without fee provided that copies are not made or distributed for profit or commercial advantage and that copies show this notice on the first page or initial screen of a display along with the full citation. Copyrights for components of this work owned by others than ACM must be honored. Abstracting with credit is permitted. To copy otherwise, to republish, to post on servers, to redistribute to lists, or to use any component of this work in other works requires prior specific permission and/or a fee. Permissions may be requested from Publications Dept., ACM, Inc., 2 Penn Plaza, Suite 701, New York, NY 10121-0701 USA, fax +1 (212) 869-0481, or permissions@acm.org.

(c) 2015 ACM 1049-3301/2015/02-ART11 $\$ 15.00$

DOI: http://dx.doi.org/10.1145/2688909 


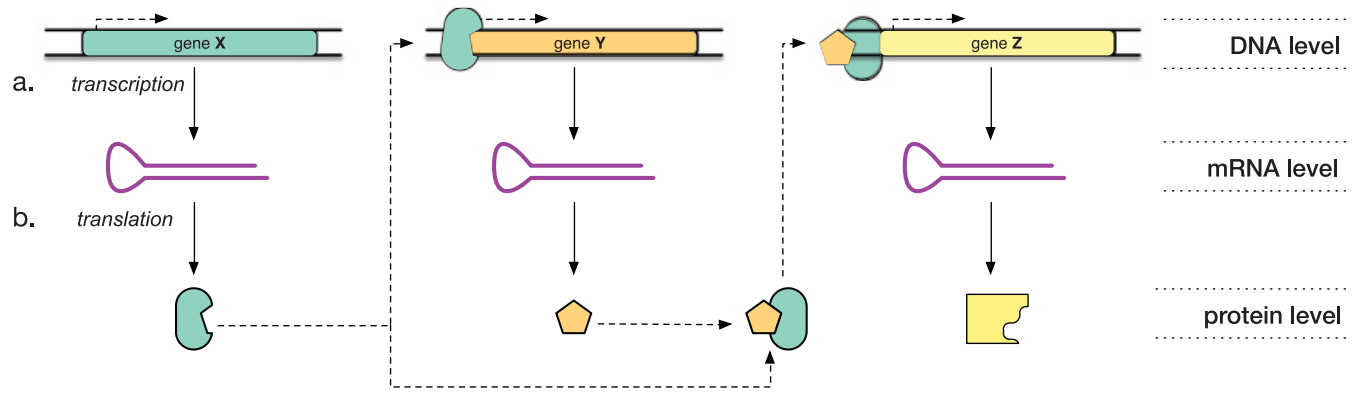

c.

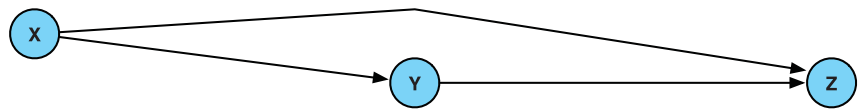

GRN

Fig. 1. Simplified representation of the regulatory mechanisms involved in a gene regulatory network. Gene $X$ regulates gene $Y$, by encoding a transcription factor which activates the transcription of gene $Y$. Genes $X$ and $Y$ coregulate gene $Z$ : The proteins produced by $X$ and $Y$ interact to form a complex, which activates gene $Z$. In the gene regulator network inference problem, one aims to reconstruct the relations between genes (bottom of the figure).

(Figure 1(a)). Next, the mRNA is translated into a sequence of amino acids, which constitute a protein (Figure 1(b)).

The products of each of these steps may be involved in the process of gene regulation. For instance, proteins called Transcription Factors (TFs) can bind directly to meaningful regions of the DNA, leading to (1) enhanced mRNA production associated with a gene, and possibly its translation into proteins, or (2) inhibition of the process associated with transcription and, hence, gene expression. Some noncoding RNA fragments-that is, RNA which is not translated into proteins-are also associated with regulation of gene expression. For instance, micro RNA (miRNA) may bind to mRNA, promoting its degradation or preventing it from being translated into proteins [Lim et al. 2005].

A detailed description of the system involving each of these regulatory mechanisms would not appear a viable option for studying cells at a system level, due to its enormous complexity. Therefore this machinery is simplified and projected onto the transcriptomic level, where only genes are considered (DNA level in Figure 1).

The set of regulatory interactions involving genes in a cell is referred to as Gene Regulatory Network (GRN). GRNs capture both transcriptomic and proteomic regulatory events (Figure 1(c)), which are implicitly encoded in the gene regulation process and difficult to interpret in physical terms. In turn, uncovering the nature of gene regulatory interactions is referred to as GRN inference and it is of central importance in systems biology. Its use is crucial in understanding system regulations and to devise effective medical interventions, and it has been shown to be very promising in understanding some genetic diseases such as cancer [Madhamshettiwar et al. 2012].

GRNs can be reconstructed from manual literature curation [Bauer-Mehren et al. 2009], or using reverse engineering computational approaches [De Jong 2002]. These two flows have different limitations. The former lacks the possibility of integrating novel measurements, for instance, in possibly compromised signaling networks, and therefore it cannot detect cellular responses under specific biological stimuli. Causal signaling links can vary depending on lineage and (epi)genetic background, such that the same perturbation can lead to different signaling responses in different backgrounds. Thus, it is important to be able to feed a prediction method with experimental data that can be acquired for the specific biological context of interest. A biological 
context may, for example, be defined by a combination of cell line (a specific type of cell) and growth condition (an external stimuli which enhances the production of some cellular product). The latter methods inherently take account of the biological context of an experiment. On the other hand, they may fail to observe well studied interactions, when the experimental data with which they are fed only includes particular cell conditions.

The development of new technologies in molecular biology (e.g., DNA microarray or high-throughput sequencing) has made available a wealth of genomic data, encouraging the development of novel computational methods for GRN inference. However, datasets are highly heterogeneous, containing information which is limited and difficult to analyze [Zhou et al. 2006]. This impacts the performance of GRN inference methods, which tend to be biased toward specific types of data [Kim et al. 2003].

To alleviate these difficulties, several alternatives have been proposed, such as methods to integrate heterogeneous data into the inference model [Sîrbu et al. 2012; Eduati et al. 2012] or to integrate a collection of predictions across different inference methods-as in the Community Network (CN) approach [Marbach et al. 2012]. Methods based on integration of heterogeneous data are a promising research direction, but they face several challenges, which span from how to relate different types of data to issues of data normalization [Sun and Zhao 2009]. The CN method has the advantage of promoting the benefits of individual methods, while smoothing out their drawbacks. CN does not exclude the use of heterogeneous data in the initial prediction set, and has been shown to be robust across species and datasets [Marbach et al. 2012]. The CN approach poses many challenges, for example, (i) how to account for strengths/weaknesses of individual methods-for example, the difficulty of mutual information methods to discriminate TFs; and (ii) how to use information not handled by the individual methods.

We propose a methodology based on Constraint Programming (CP) to combine community predictions and integrate biological knowledge-leading to a new paradigm for $\mathrm{CNs}$, referred to as constrained community networks. $\mathrm{CP}$ is a declarative problem solving paradigm, where logical rules are used to model problem properties and to guide the construction of solutions. $\mathrm{CP}$ offers a natural environment where heterogeneous information can be actively handled. The use of constraint expressions allows the incremental refinements of a model. This is particularly suitable to model biological knowledge integration, when such knowledge cannot be directly handled by individual prediction methods. CP provides an effective framework to model different types of network information that may become available during problem modeling or hypotheses testing, and use such information in the inference process.

We tested our method on a set of 360 benchmarks, including large networks proposed by the DREAM3 [Prill et al. 2010] and DREAM4 [Greenfield et al. 2010] challenges. We perform our experiments with three types of data obtained in two different experimental setups. We show significant improvements in prediction accuracy compared to a state-of-the-art CN-based approach, up to $29.5 \%$, when the integration of knowledge about target networks acquired in biological relevant settings is applied.

\section{BACKGROUND}

\subsection{Constraint Programming}

$\mathrm{CP}$ is a declarative programming paradigm commonly used to address combinatorial search problems. It focuses on capturing properties of the problem in terms of variables (representing the unknowns of the problem) and constraints over the variables (i.e., relations among the components of the problem), which are satisfied exclusively by solutions of the problem. Solutions to the problem are represented by assignments of values to the variables. CP models are fully declarative and elaboration tolerant, 
enabling the incremental integration of new knowledge and the use of sophisticated problem solving techniques (e.g., propagation and filtering methods, search heuristics).

A Constraint Satisfaction Problem (CSP) is formalized as a triple $\langle\mathbf{X}, \mathbf{D}, \mathbf{C}\rangle . \mathbf{X}=$ $\left\langle x_{1}, \ldots, x_{n}\right\rangle$ is an $n$-tuple of variables - that is, the unknowns of the problem. $\mathbf{D}=$ $\left\langle D_{1}, \ldots, D_{n}\right\rangle$ is a corresponding $n$-tuple of domains; each $D_{i}$ is a set of values, specifically the admissible values for the variable $x_{i} . \mathbf{C}=\left\langle C_{1}, \ldots, C_{k}\right\rangle$ is a $k$-tuple of constraints. Let us consider a subset $S_{j} \subseteq \mathbf{X}$ of the variables; a constraint $C_{j}$ over $S_{j}$ is a subset of the Cartesian product of the domains of the variables in $S_{j}$-that is, $C_{j} \subseteq \Pi_{x_{r} \in S_{j}} D_{r}$. Intuitively, a constraint over the variables $S_{j}$ restricts what are the joint assignments of values to the variables in $S_{j}$.

Given an $n$-tuple $A=\left\langle a_{1}, \ldots, a_{n}\right\rangle \in D_{1} \times \cdots \times D_{n}$, we denote with $\left.A\right|_{S_{j}}$ the projection of the tuple on the variables in $S_{j}$. For example, if $S_{j}=\left\{x_{1}, x_{2}\right\}$, then $\left.A\right|_{S_{j}}=\left\langle a_{1}, a_{2}\right\rangle$. The largest (smallest, respectively) value that can be assigned to a variable $x_{i}$ is denoted by $\max \left(D_{i}\right)\left(\min \left(D_{i}\right)\right.$, respectively).

A solution of a $\operatorname{CSP}\langle\mathbf{X}, \mathbf{D}, \mathbf{C}\rangle$ is an $n$-tuple $A=\left\langle a_{1}, \ldots, a_{n}\right\rangle$, where $a_{i} \in D_{i}($ for $1 \leq i \leq n)$ and $\left.A\right|_{S_{j}} \in C_{j}$ (for $1 \leq j \leq k$ ) -that is, the projection of $A$ on the set of variables involved in $C_{j}$ satisfies the relation $C_{j}$. Typical resolution algorithms for CSPs rely on efficient search procedures, to explore the space of possible solutions, and on consistency methods, where constraints are used to remove infeasible elements from the domains of not yet assigned variables. This search is made by exploring a data structure called prop-labeling-tree [Apt 2009] composed by two kind of nodes: (i) nodes with as many children as the current size of the domain of a selected variable (nondeterministic choices) and (ii) nodes with a unique child obtained deterministically by a process of constraint propagation. Search strategies are developed for alternative visiting of the search tree (that is dynamically computed using backtracking). Incomplete methods are used for large problems, where the search is guided by random choices.

\subsection{Gene Regulatory Networks and Inference Methods}

A Gene Regulatory Network (GRN) can be described by a weighted directed graph $\mathcal{G}=$ $(V, E)$, where $V$ is the set of regulatory elements of the network and $E \subseteq V \times V \times[0,1]$ is the set of regulatory interactions. The presence of an edge $\langle s, t, w\rangle \in \bar{E}$ indicates that an interaction between the regulatory elements $s$ and $t$ is present with confidence value $w \in[0,1] \subseteq \mathbb{R}$. The number $|V|$ is referred to as the size of the GRN. If the GRN has no uncertainty, then each edge in $E$ will have weight equal to 1.

In the problem of GRN inference, we are given the set of vertices $V$ (in this article each $g_{i} \in V$ represents a gene) and a set of experiments, describing the behavior of the regulatory elements. The goal is to accurately detect the set of regulatory interactions $E$. The observations associated with the expression profiles of the gene $g_{i}$ in a GRN are described via a random variable $G_{i}$ whose values are typically normalized in $[0,1]$.

We provide an overview of the network inference methods adopted in our investigation. We classify methods in five classes according to their main component.

2.2.1. Correlation. Correlation-based network inference methods rely on the notion of statistical dependence, a condition in which random variables do not satisfy a requirement of probabilistic independence. The correlation between gene expression levels is expressed by a number in $[-1,1] \subseteq \mathbb{R}$ to indicate the presence of a regulatory interaction. A positive (negative) value indicates an activating (inhibitory) interaction. We consider three standard correlation coefficiens: Pearson, Spearman, and Kendall. Pearson coefficient relates the standard deviation of the expression profiles of two genes with their covariance and it is limited to capture linear dependencies. The other two relate the ranked expression levels of genes and can capture how well two variables can be described via a monotonic function. The correlation coefficients considered are 
symmetric: that is, for two random variables $X$ and $Y$ it holds that $\operatorname{corr}(X, Y)=$ $\operatorname{corr}(Y, X)$. Therefore, additional information is required to assign directionality to the inferred interactions. As our evaluation does not discriminate inhibiting from activating interactions, we focus on the absolute value of the correlation coefficients. ${ }^{1}$ Correlation measures are widely adopted in practice to study the relationships among gene expressions, for example, in D'Haeseleer et al. [1999] they have been used to reconstruct the GRN associated with the central nervous system development in rats.

2.2.2. Mutual Information. One of the limitations of correlation-based methods is their inability to identify nonlinear relationships among variables. Mutual Information (MI) methods overcome this limitation by measuring the common information in two random variables $X$ and $Y$. Let us assume that $X(Y)$ range on a finite set $\mathcal{D}_{X}\left(\mathcal{D}_{Y}\right)$. In a GRN inference context these values emerge from a discretization of the expression levels of the associated genes emerging from experimental measurements, possibly normalized in $[0,1]$. This approach quantifies to which extent knowing one of these variables reduces the uncertainty about the other. For instance, if $X$ and $Y$ are independent, no additional information about $Y$ is produced by knowing $X$ and vice versa; thus, their MI is 0 . We denote with $I(X ; Y)$ the MI of variables $X$ and $Y$. MI-based methods cannot infer the direction of an interaction.

The Context Likelihood of Relatedness (CLR) [Faith et al. 2007] assigns a score $z_{i j}$ to each interaction between genes $g_{i}, g_{j}: z_{i j}=\sqrt{z_{i}^{2}+z_{j}^{2}}$, with $z_{i}=\max _{j \neq i}\left(0, \frac{I\left(G_{i} ; G_{j}\right)-\mu_{i}}{\sigma_{i}}\right)$ where $\mu_{i}$ and $\sigma_{i}$ are, respectively, the mean and the standard deviation of the empirical distribution of the MI values $I\left(G_{i} ; G_{k}\right)$ of $G_{i}$ for all the variables $G_{k}, k \neq i$. This represents the background distribution of the MI for gene $g_{i}$ and it plays a central role in the CLR algorithm by aiming at reducing the prediction of false interactions-based on false correlations-and indirect interactions. CLR has been successfully applied to decipher the Escherichia coli transcriptional regulatory network [Faith et al. 2007].

The Algorithm for Reconstruction of Accurate Cellular Networks (ARACNE) [Margolin et al. 2006] aims at filtering out indirect interactions by applying the Data Processing Inequality (DPI). The DPI states that if gene $g_{i}$ interacts with gene $g_{j}$ through a gene $g_{k}$ then $I\left(G_{i} ; G_{j}\right) \leq \min \left[I\left(G_{i} ; G_{k}\right), I\left(G_{j} ; G_{k}\right)\right]$. After computing the MI of the pair of genes involved, ARACNE filters out all the interactions for which their MI does not exceed a given threshold. Then it prunes the weakest interactions within each triplet of genes if it violates the DPI test. This approach has been validated using microarray dataset from reconstructing the GRN associated with human B cells [Margolin et al. 2006].

The Conservative Causal Core (C3NET) [Altay and Streib 2010] algorithm consists of two steps. First, it detects the nonsignificant connections among gene pairs $g_{i}, g_{j}$. This is realized by testing the statistical significance of their MI $I\left(G_{i} ; G_{j}\right)$, by assessing whether the null hypothesis $H_{0}: I\left(G_{i} ; G_{j}\right)=0$ cannot be rejected for a given significance level. For each gene $g_{i}$, it selects the most significant link $\left(g_{i}, g_{j}\right)$ for which the null hypothesis cannot be rejected based on their MI estimate.

$\underline{B C 3 N E T}$ is an extension of C3NET in which an ensemble of datasets is generated via bootstrapping and each of the bootstrapped datasets is fed to the C3NET network inference procedure. The inferred networks are hence aggregated employing a binomial test [de Matos Simoes and Emmert-Streib 2012]. A C3NET algorithm has been used to identify tumor-specific gene interactions in prostate cancer datasets [Altay et al. 2011].

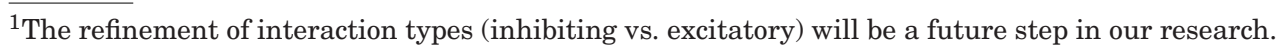


2.2.3. Other Statistical Tests. The Generalized Logical Network (GLN) models interactions as many-to-one relationships between a set of TFs and a target gene [Song et al. 2009]. An interaction is ranked by its $p$ value in the $\chi^{2}$ test; in the case of ties, interactions with lower degrees of freedom are ranked higher. The significance of the $\chi^{2}$ statistics accounts for both linear and nonlinear interactions. GLN has been adopted to identify genes from major neuronal pathways in the alcohol response mechanism from the brains of alcohol-treated mice [Song et al. 2009].

2.2.4. Feature Selection. In the context of supervised learning, feature selection is the process of selecting a subset of relevant features to be used in the model construction. This process can be viewed as an optimization problem, where the measure to be optimized is a score of the different subsets of features. Since the general problem of selecting the best subset of features is computationally intractable, several techniques based on (incomplete) local search methods are commonly adopted [Hastie et al. 2009].

MRNET infers interactions between genes by using MI between expression profiles and a feature selection procedure called Maximum Relevance Minimum Redundancy (MRMR) [Meyer et al. 2007]. For each variable $G_{j}$, treated as a target gene, MRNET aims at selecting a set of regulators $\mathbf{S}_{j}$ of $g_{j}$ having high MI with $G_{j}$ (maximum relevance) and low MI between them (minimum redundancy). The selection of the set $\mathbf{S}_{j}$ is made via a forward selection procedure, which starts by including the variable with the highest MI with the target $G_{j}$. The other variables being selected will be the ones having high MI with $G_{j}$ and low MI with the variables already in $\mathbf{S}_{j}$. A specific network can then be inferred by only keeping edges whose score lies above a given threshold (similarly to what is done in CLR).

Gene Network Inference with Ensemble of Trees (GENIE3) is similar to MRNET, in that (a) it considers each gene individually, treating it as the target gene regulated by the other genes, and (b) it employs a feature selection procedure to identify the best set of regulator genes. GENIE3 uses a decision tree learning approach, where leaves nodes of the decision tree describe class labels, while each internal node represents a test on an attribute, and each branch represents the outcome of a test. A path from the root to a leaf node represents a classification rule. A decision tree can be learned by splitting the set of items into subsets based on an attribute value test, so to create different branches, and repeating this process recursively on each derived subset.

The feature selection step of GENIE3 is performed via random forests [Breiman et al. 1984]. At each feature selection step, GENIE3 generates an ensemble of 1,000 trees, built using a bootstrap sample composed of $p-1$ randomly selected attributes, where $p$ is the number of potential regulators. In each tree each node $n$ selected for a split is augmented with a score that accounts for the total reduction of the variance of the output variable due to the split: $I(n)=|\mathbf{S}| \sigma(\mathbf{S})-\left|\mathbf{S}_{t}\right| \sigma\left(\mathbf{S}_{t}\right)-\left|\mathbf{S}_{f}\right|$, where $\mathbf{S}$ denotes the set of samples reaching node $n, \mathbf{S}_{t}$ and $\mathbf{S}_{f}$ denote the subsets of $\mathbf{S}$ for which the test was respectively true or false, and $\sigma(\cdot)$ is the variance of the output variable in a given set. For each target gene, the importance of a gene as its regulator is computed by summing the tree nodes where such gene is used as a variable to split the tree, and averaging the results across the ensemble of trees. The results of each subproblem are aggregated to get the final ranked list of regulatory interactions. GENIE3 was the best performer in the DREAM4 challenge [Greenfield et al. 2010].

Tigress, similarly to MRNET and GENIE3, employs a feature selection strategy to estimate a score $s_{j}(i)$ of each candidate regulator $g_{i}$ for a target gene $g_{j}$. This is determined as the solution of a regression problem, aimed at predicting the expression level $G_{j}$ from the expression level of its candidate regulators $G_{i} \in \mathbf{S}_{j} \subseteq \mathbf{G}$ : $G_{j}=f_{j}\left(\mathbf{S}_{j}\right)+\epsilon$, where $\mathbf{G}$ is the set of all the $G_{i}$ 's associated with the genes of the GRN, 
$f_{j}$ is a regression function, and $\epsilon$ is a term modeling some noise. The algorithm does not aim to model the regression function $f_{j}$, but rather to find a small set of regulators $\mathbf{S}_{j}$ which are sufficient to provide a good model for $G_{j}$. The score $s_{j}(i)$ is associated with each candidate regulator $g_{i}$, and it assesses the likelihood of $G_{i}$ to be involved in the regression model $f_{j}$; this is computed via a Least Angle Regression (LAR) [Efron et al. 2004] with stability selection [Meinshausen and Bühlmann 2010]. Tigress was evaluated to be the best linear regression-based method in the DREAM5 gene network inference challenge [Marbach et al. 2012] and one among the top overall performers.

2.2.5. Meta Approaches. The inferelator pipeline is a meta approach based on resampling combining Median-Corrected z-Scores (MCZ), to rank edges based on a z-score derived from TF-deletion data, Time-Lagged CLR (tlCLR), for the analysis of timeseries data, and a linear ordinary differential equation (ODE) model constrained by Lasso [Greenfield et al. 2010]. The kernel of the inferelator is based on a ODE model which governs the time evolution of a gene product accounting for both RNA production and degradation rates for each gene (see Bonneau et al. [2006] for details).

The inferelator was used to predict a large portion of the regulatory network of the archaeon Halobacterium NRC-1 under specific perturbations [Bonneau et al. 2006].

2.2.6. Community Inference as Committees. Combining different models for solving classification problems has been an active topic of research in machine learning [Bishop and Nasrabadi 2006; Renda and Straccia 2003]. The use of multiple trained models in combination often results in improved performance and enhanced robustness with respect to merely using a single model in isolation [Renda and Straccia 2003]. A widely adopted aggregation strategy is that of committees, a metapredictor where multiple models are combined by averaging the results of each individual predictor.

Consider a simple regression problem where we want to predict the value of a continuous function $t(x)$. The committee prediction for an input $x$ is given by averaging the predictions of the $M$ committee members: $\hat{t}(x)=\frac{1}{M} \sum_{i=1}^{M} f_{i}(x)$, where $f_{i}(x)$ is the prediction of the $i$ th method in the committee at input $x$. If the prediction errors made by the individual predictors are all uncorrelated and have 0 mean, the average error of a model could be reduced by a factor of $M$ simply by averaging $M$ members. Even though typically errors are highly correlated and the performance gain could be small, the work of Perrone [Perrone 1993] shows that even when committee members are correlated and biased, the squared prediction error of the committee (obtained through an averaging process) is no worse than the mean squared prediction error of the individual committee members, that is, $(\hat{t}(x)-t(x))^{2} \leq \frac{1}{M} \sum_{i=1}^{M}\left(f_{i}(x)-t(x)\right) .^{2}$ Informally, this means that an averaging process can only improve the results, provided that the committee members make better than random predictions.

In a binary classification problem, $t(x)$ could be interpreted as the probability of belonging to one class (e.g., the presence of a regulatory relation), and $1-t(x)$ as the probability of belonging to the other class (e.g., the absence of a regulatory relation); $f_{i}(x)$ represents the prediction of the $i$ th method for $t(x)$.

In the context of GRN inference, committees are referred to as Community Networks $(\mathrm{CNs})$ and are used to integrate multiple inference methods to obtain a common consensus prediction, as illustrated in Figure 2, where the $P_{i}$ 's represent the GRN predictions obtained by different methods. CNs have been shown to achieve better average confidence across different datasets and produce more robust results with respect to the individual methods being composed [Marbach et al. 2012]. A simple scheme for combining predictions in a CN has been proposed by Marbach et al. [2012] where each interaction is rescored by averaging the ranks it obtained within each of all the employed predictions: We will refer to this method as $\mathrm{CN}_{\text {rank }}$.

The inference methods adopted in this study are listed in Table I. 


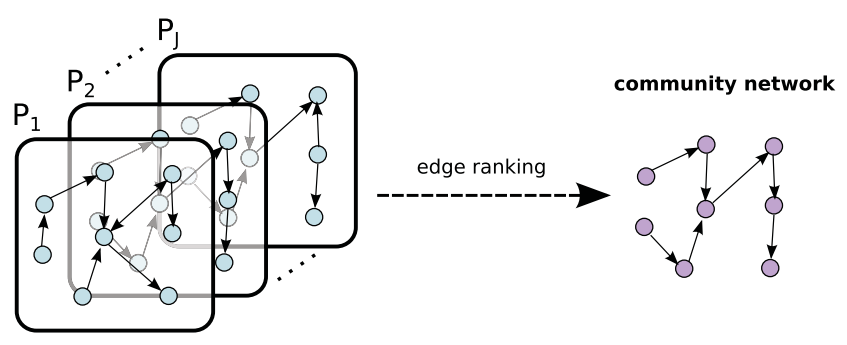

Fig. 2. CN generation via committee models integration.

Table I. The CN Prediction Methods Adopted

\begin{tabular}{|lccccc|}
\hline Pearson & Spearman & Kendall & MRNET & Aracne & CLR \\
C3Net & BC3Net & Tigress & Genie3 & Inferelator & GLN \\
\hline
\end{tabular}

\section{CONSTRAINED COMMUNITY GRN INFERENCE}

Constraint technologies and Constraint Programming (CP) have been recently successfully applied in the field of system biology [Videla et al. 2012]. For example, answer set programming has been adopted to address problems in network inconsistencies detection [Gebser et al. 2008] and in metabolic network analysis [Soh and Inoue 2010]. CP has been used to investigate discrete network models, under Thomas' GRN model [Thomas 1973], where GRNs are modeled using multivalued variables and transition rules [Corblin et al. 2009]. In particular, CP is used to represent GRNs' possible dynamics and to test, for a given structure of a GRN, the consistency of a set of hypotheses-allowing the relaxation of the constraints imposed on the network behavior when inconsistencies arise [Corblin et al. 2010]. The Biocham platform [Fages et al. 2010] makes use of temporal and other classes of constraints to support modeling and simulation of regulatory networks. Concurrent constraint programming has also been used to support modeling of biological systems [Bortolussi and Policriti 2008], where interacting molecules are viewed through the lenses of communicating processes.

The CN approach adopted in this work is built by combining multiple GRN inference procedures and creating an inference ensemble. The methods used to create the ensemble have been selected based on their performance, popularity, and availability.

The methods selection process used to build an ensemble starts from a set of 12 methods from the classes described in Section 2.2. While building the final ensemble, we impose the constraint that exactly one representative method from each class should be an ensemble component; the only exception is when two methods in a class are distinguished by a secondary component. This choice provides robustness and diversity, while avoiding redundancies that could potentially bias the inference ensemble.

A preliminary version of the research described in this article has been presented in Fioretto and Pontelli [2013]. The work presented in this article provides a more detailed model description and formalization of the CN-based GRN inference problem, introducing a new set of constraints, more general and effective than those originally discussed in Fioretto and Pontelli [2013]. The present work also removes several restrictive assumptions used in our previous work by limiting, analyzing, and automatically tuning the constraints' parameters. In addition, while our previous work restricted the constraint solver to integer finite domains (and therefore created the potential for discretization errors), the constraint solver introduced in this article is capable of handling real values. The present article introduces a comprehensive assessment of the CN-based GRN inference schema. It includes an extensive evaluation of a broad set of 
individual prediction methods and their combination in committees, and analyzes the proposed method on a wide set of large GRNs and three different datasets.

\subsection{Problem Formulation}

Given a set of $n$ genes, we describe a GRN inference problem as a $\operatorname{CSP}\langle\mathbf{X}, \mathbf{D}, \mathbf{C}\rangle$, where $-\mathbf{X}$ is a set of $n^{2}-n$ variables, each of them referred to as $X_{i \rightarrow j}$, with $i, j \in\{1, \ldots, n\}$, $i \neq j$. These variables describe regulatory relations (excluding self-regulations);

-D is the set of domains for the variables in $\mathbf{X}$. Each $D_{i \rightarrow j}$ is a finite set of elements in $[0,1] \subset \mathbb{R}$, describing the possible confidence values associated with the regulatory relation modeled by $X_{i \rightarrow j}$. Values close to 0 indicate high confidence about the absence of a regulatory relation (with 0 denoting the highest confidence), whereas values close to 1 indicate high confidence about the presence of a regulatory relation (with 1 denoting the highest confidence); and

- $\mathbf{C}$ is a $k$-tuple of constraints $\left\langle C_{1}, \ldots, C_{k}\right\rangle$. Each $C_{j}$ is a constraint over a set of variables $S_{j} \subseteq \mathbf{X}$. Constraints expressing restrictions of peculiar network topologies will be discussed in Section 3.3.

A variable $X_{i \rightarrow j}$ is said to be assigned when its associated domain $D_{i \rightarrow j}$ is a singleton. We adopt the notation $d\left(X_{i \rightarrow j}\right)$ to indicate the value of an assigned variable $X_{i \rightarrow j}$. A solution to the preceding CSP defines a GRN prediction $G=(V, E)$, with $V=\{1, \ldots, n\}$ and $E=\left\{\left\langle i, j, d\left(X_{i \rightarrow j}\right)\right\rangle \mid i \in V, j \in V, d\left(X_{i \rightarrow j}\right)>0\right\}$.

\subsection{Ensemble Analysis and Initial Domains Construction}

The proposed CSP solution leverages the collection of GRN predictions within the prediction ensemble by (1) tightening the size of the solution search space ${ }^{2}$ and (2) taking into account the discrepancies among the community predictions. These objectives are achieved by mapping the edge confidence levels of each prediction to the corresponding CSP variable domain. The greater the agreement in the inference ensemble, the smaller is the set of values in the domain of the variable representing the relation being considered. Thus, the size of each domain captures the degree of uncertainty expressed by an edge prediction within the inference ensemble.

Let us consider a set of $J$ predictions $\mathcal{P}$ of a GRN $G=(V, E)$. For $j \in\{1, \ldots, J\}$, let us denote with $P_{j}=\left(V, E_{j}\right)$ the $j$ th prediction, where $E_{j}$ are the edges that have been identified by $P_{j}$. For a labeled edge $(s, t, w) \in E_{j}$ we identify $w$ as the confidence assigned for the presence of that edge in the GRN, and $\omega_{j}^{\#}(s, t)$ as its rank-its position in the descending ordered list of confidence values of $E_{j}$-normalized in $[0,1]$, where 0 is associated with the last position, and 1 to the first one. Furthermore, let $\theta_{d}\left(0 \leq \theta_{d} \leq 1\right)$ be a given threshold, referred to as the disagreement threshold.

The procedure described in Algorithm 1 populates the domains in $\mathbf{D}$ with at most three values. For each edge $(s, t)$, we calculate (line 4$)$ the average confidence value w_rank, according to the Borda count election method, as presented by Marbach et al. [2012]. This method averages the ranked edge confidence values $\left[\omega_{j}^{\#}(s, t)\right]$ assigned by each prediction $P_{j}$. Line 4 also determines the discrepancy value w_d within $\mathcal{P}$. The discrepancy value captures the ensemble prediction disagreement for a given edge, averaging the pairwise differences of the edge ranks associated with each prediction of the ensemble. If the discrepancy value exceeds the discrepancy threshold $\theta_{d}$ and the average confidence value is not strongly informative-that is, it lies between values $L \in[0,1]$ and $U \in[0,1]$ (line 6)-then we force the domain $D_{s \rightarrow t}$ to account for the prediction disagreement by adding a variation of $\mathrm{w}_{-} \mathrm{d} / 2$ to the average confidence value.

${ }^{2}$ For domains having all size $b$ the search space of a GRN inference problem of size $n$ is $b^{n^{2}-n}$. 


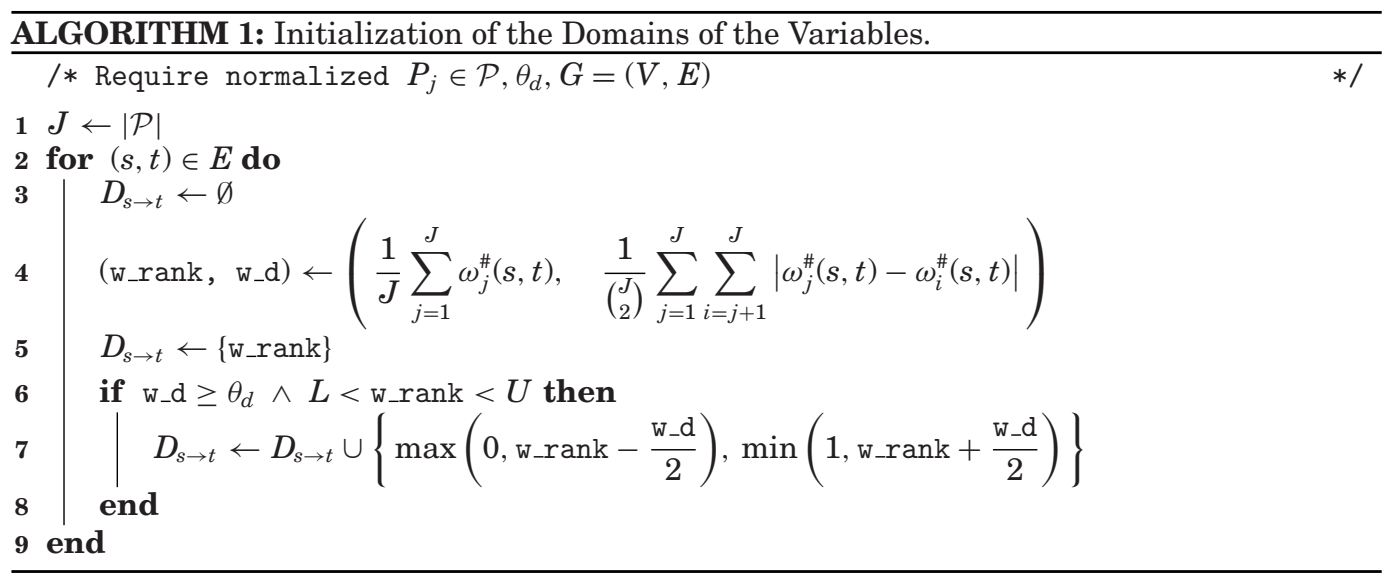

Line 5 ensures the presence of the value w_rank in $D_{s \rightarrow t}$. All the parameters of the algorithm-that is, $\theta_{d}, L$, and $U$-are automatically tuned, and they depend entirely on the prediction ensemble (see Section 4.3.1 for details).

\subsection{Constraints}

3.3.1. Sparsity Constraints. It is widely accepted that the GRN machinery is controlled by a relatively small number of genes. Several state-of-the-art methods for predicting GRNs encourage sparsity in the inferred networks [Marbach et al. 2012]. Nevertheless, when combining predictions in a community-based approach, no guarantees on the sparsity of the resulting prediction can be provided. To address this issue, we introduce a sparsity constraint, which is built from two more general constraints: atleast_k_ge and atmost_k_ge. They both enforce a relation among a set of variables, to ensure that at least (at most, respectively) $k$ of the variables have values exceeding a given threshold:

$$
\text { atleast_k_ge }(k, \mathbf{S}, \theta) \equiv\left|\left\{X_{i} \in \mathbf{S} \mid d\left(X_{i}\right)>\theta\right\}\right| \geq k .
$$

Equation (1) enforces a lower bound $(k \in \mathbb{N})$ on the number of variables in $\mathbf{S} \subseteq \mathbf{X}$ whose confidence value is greater than $\theta$ (with $0 \leq \theta \leq 1$ ). The constraint atmost_k_ge is defined in the same way where $\geq k$ is replaced by $\leq k$.

These constraints are used to filter the domains of the variables involved, through a propagation process. The propagation of the atmost_k_ge constraint is exploited during solution search to enforce its semantics and performed by the following rewriting rule: ${ }^{3}$

$$
\text { atmost_k_ge }(k, \mathbf{S}, \theta): \frac{\mathbf{T}=\left\{X_{i} \in \mathbf{S} \mid \min \left(D_{i}\right)>\theta\right\},|\mathbf{T}|=k}{\bigwedge_{X_{i} \in \mathbf{S} \backslash \mathbf{T}} D_{i}=D_{i} \cap[0, \theta]} .
$$

Intuitively, if there are already $k$ variables in $\mathbf{S}$ whose possible values are greater than $\theta$, then all other variables should have $\theta$ as an upper bound to their admissible value.

For the atleast_k_ge constraint, early failures can be detected during the solution search, by checking the upper bound on the number of variables not yet instantiated

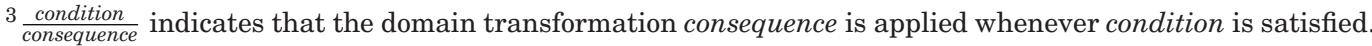




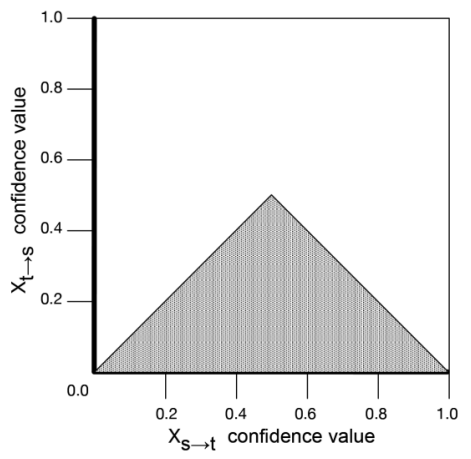

Fig. 3. Values for $X_{t \rightarrow s}$.

which satisfy property (1). The associated propagation rule is

$$
\text { atleast_k_ge }(k, \mathbf{S}, \theta): \frac{\mathbf{T}=\left\{X_{i} \in \mathbf{S} \mid \max \left(D_{i}\right) \leq \theta\right\},|\mathbf{S} \backslash \mathbf{T}|=k}{\bigwedge_{X_{i} \in \mathbf{S} \backslash \mathbf{T}} D_{i}=D_{i} \cap(\theta, 1]} .
$$

The sparsity constraint ranges over the variables in $\mathbf{X}$. It enforces lower and upper bounds on the number of edges whose confidence value is outside a given threshold. Formally, given $k_{l}, k_{m}, \theta_{l}, \theta_{m}$ (these parameters are discussed in Section 4.3.1):

$$
\operatorname{sparsity}\left(\mathbf{X}, k_{l}, \theta_{l}, k_{m}, \theta_{m}\right) \equiv \text { atleast_k_ge }\left(k_{l}, \mathbf{X}, \theta_{l}\right) \cap \text { atmost_k_ge }\left(k_{m}, \mathbf{X}, \theta_{m}\right) \text {. }
$$

3.3.2. Edge Orientation Constraint. Given two variables $X_{s \rightarrow t}$ and $X_{t \rightarrow s}$ the edge orientation constraint (orient) exploits the confidence value assigned to $X_{s \rightarrow t}$ to impose an upper bound on the values that can be assigned to $X_{t \rightarrow s}$. This constraint imposes an orientation for an edge between two given nodes and it is described as follows:

$$
\operatorname{orient}\left(X_{s \rightarrow t}, X_{t \rightarrow s}\right) \equiv X_{t \rightarrow s} \leq \min \left(X_{s \rightarrow t}, 1-X_{s \rightarrow t}\right) \text {. }
$$

This constraint bounds the variable $X_{t \rightarrow s}$ to the confidence value of $X_{s \rightarrow t}$ and, if the existence of the edge $(s, t)$ is predicted with a high confidence $(>0.5)$, by a factor which is inversely proportional to $X_{s \rightarrow t}$. Figure 3 depicts the upper bound for the confidence values of the variable $X_{t \rightarrow s}$ (the solid line) at the varying of the values of the variable $X_{s \rightarrow t}(x$ axis).

The propagation of the orient constraint is exploited during the solution search to enforce property (5) and implemented by the rule

$$
\operatorname{orient}\left(X_{s \rightarrow t}, X_{t \rightarrow s}\right): \frac{v=d\left(X_{s \rightarrow t}\right), l=\min (v, 1-v)}{D_{t \rightarrow s}=D_{t \rightarrow s} \cap[0, l)} .
$$

3.3.3. Redundant Edge Constraints. Several state-of-the-art inference methods rely on MI or correlation techniques. The community approach adopted in this work employs methods that use both correlation and MI as principal components for the inference process. One of the disadvantages of such methods is the difficulty in speculating on the directionality of a given prediction. We define a constraint that can aid in detecting the edge directionality based on the collective decisions of the $\mathrm{CN}$ predictions, among the non MI- or correlation-based methods.

Let us consider a collection of predictions $\mathcal{P}=\left\{P_{1}, \ldots, P_{J}\right\}$ for a GRN $G=(V, E)$, and a nonempty set of predictions $\mathcal{H} \subseteq \mathcal{P}$ derived from MI-based or correlation-based methods. An edge $(t, s)$ is said to be redundant if

$$
\forall P_{i} \in \mathcal{P} \backslash \mathcal{H} . \quad \omega_{i}(s, t)>\omega_{i}(t, s)+\beta_{i},
$$


where $\omega_{i}(s, t): V \times V \rightarrow[0,1]$ expresses the confidence value of the edge $(s, t)$ in the prediction $P_{i}$, and $\beta_{i} \in \mathbb{R}$ is a real value associated with each prediction method in $\mathcal{H}$. Similarly, we define a variable $X_{s \rightarrow t}$ to be redundant if the corresponding edge $(s, t)$ is redundant. We use the proposition red-e $(t, s)$ to denote a redundant edge $(t, s)$, and given a redundant edge $(t, s)$ we call the edge $(s, t)$ the required edge. The redundant_edge constraint enforces a relation between two variables $X_{s \rightarrow t}$ and $X_{t \rightarrow s}$ by imposing an edge orientation constraint on the redundant variable and the required variable. Let $X_{R}$ be the set of all the required and redundant variables. ${ }^{4}$ For a pair of variables $X_{s \rightarrow t}, X_{t \rightarrow s} \in X_{R}$ we express a redundant edge constraint as

$$
\text { redundant }\left(X_{s \rightarrow t}, X_{t \rightarrow s}\right) \equiv \operatorname{red-e}(t, s) \rightarrow \operatorname{orient}\left(X_{s \rightarrow t}, X_{t \rightarrow s}\right),
$$

which naturally translates to the propagation rule:

$$
\text { redundant }\left(X_{s \rightarrow t}, X_{t \rightarrow s}\right): \frac{v=d\left(X_{s \rightarrow t}\right), l=\min (v, 1-v), \operatorname{red}-\mathrm{e}(t, s)}{D_{t \rightarrow s}=D_{t \rightarrow s} \cap[0, l)} .
$$

3.3.4. Transcription Factor Constraint. GRN-specific information, for example, sequence DNA-binding TFs or functional activity of a set of genes, is often available from public sources (e.g., DBD [Kummerfeld and Teichmann 2006] or Gene Ontology [Harris et al. 2004]). Moreover, several studies show that similar mRNA expression profiles are likely to be regulated via the same mechanisms [Allocco et al. 2004]. On the other hand, not every method may be designed to handle such information, or this information may be only partially available, and hence not suitably usable by prediction methods. We propose constraints to incorporate such information in the CN model.

The property that a TF regulates the production of other genes is described by a condition on the out-degree of the involved gene-for those edges with an adequate confidence value. The $t$-factor constraint over a gene $s$ requires the condition: atleast_k_ge $\left(k, \mathbf{X}_{s}, \theta\right)$, with $\mathbf{X}_{s}=\left\{X_{s \rightarrow u} \in \mathbf{X} \mid u \in V\right\}$, and $k$ represents the coexpression degree, that is, the number of genes targeted by the TF. In addition, we impose a directionality constraint between each variable $X_{s \rightarrow u}$ and $X_{u \rightarrow s}$ whenever $u$ has not been identified as a TF:

$$
\text { t-factor }(s, k, \theta) \equiv \text { atleast_k_ge }\left(k, \mathbf{X}_{s}, \theta\right) \cap \bigcap_{u \in X_{s} \backslash \mathbf{T F}} \operatorname{orient}\left(X_{s \rightarrow u}, X_{u \rightarrow s}\right),
$$

where $\mathbf{T F}$ is the set of all the putative transcription factors for the predicted GRN.

3.3.5. Coregulator Constraint. Multiple TFs can cooperate to regulate the transcription of specific genes; these are referred to as coregulators. When this information is available, it can be expressed by a co-reg constraint. This constraint involves two TFs, $s^{\prime}$ and $s^{\prime \prime}$. The constraint enforces a relation on a set of variables $\mathbf{X}_{\mathbf{S}}$, to guarantee the existence of at least $k$ elements that are coregulated by both $s^{\prime}$ and $s^{\prime \prime}$, for which an interaction is predicted with confidence values greater than $\theta(0<\theta \leq 1)$.

Given two distinct TFs $s^{\prime}, s^{\prime \prime}$ and a threshold $\theta$, the set of all elements coregulated by both $s^{\prime}$ and $s^{\prime \prime}$ is defined as follows:

$$
\mathbf{C R}_{\left\langle s^{\prime}, s^{\prime \prime}, \theta\right\rangle}=\left\{t \mid t \in V, t \neq s^{\prime}, t \neq s^{\prime \prime}, d\left(X_{s^{\prime} \rightarrow t}\right)>\theta, d\left(X_{s^{\prime \prime} \rightarrow t}\right)>\theta\right\} .
$$

As for the t-factor constraint, a directionality constraint is imposed between the transcription regulators $s^{\prime}$ and $s^{\prime \prime}$ and each of their targets $u \in \mathbf{X}_{\mathbf{s}} \backslash \mathbf{T F}$. Given a coregulation degree $k$, a real value $\theta \in(0,1]$ and a set of variables $\mathbf{X}_{\mathbf{S}}=\left\{X_{s^{\prime} \rightarrow t}, X_{s^{\prime \prime} \rightarrow t}\right\}$, for some $s^{\prime}, s^{\prime \prime}, t \in V$ different among each other and such that whenever both $X_{s^{\prime} \rightarrow t}$ and

${ }^{4} X_{s \rightarrow t}$ is required/redundant if the corresponding edge $(s, t)$ is required/redundant. 
$X_{s^{\prime \prime} \rightarrow t}$ are in $\mathbf{X}_{\mathbf{S}}$, then $X_{t \rightarrow u} \notin \mathbf{X}_{\mathbf{S}}$, for all $u \in V$, the coregulator constraint is expressed by

$$
\begin{aligned}
& \operatorname{co-reg}\left(s^{\prime}, s^{\prime \prime}, k, \mathbf{X}_{\mathbf{S}}, \theta\right) \\
& \equiv\left|\left\{\left\langle X_{s^{\prime} \rightarrow t}, X_{s^{\prime \prime} \rightarrow t}\right\rangle \mid X_{s^{\prime} \rightarrow t} \in \mathbf{X}_{\mathbf{S}}, X_{s^{\prime \prime} \rightarrow t} \in \mathbf{X}_{\mathbf{S}}, t \in \mathbf{C R}_{\left\langle s^{\prime}, s^{\prime \prime}, \theta\right\rangle}\right\}\right|>k \\
& \cap \bigcap_{u \in X_{s} \backslash \mathbf{T F}} \operatorname{orient}\left(X_{s^{\prime} \rightarrow u}, X_{u \rightarrow s^{\prime}}\right) \cap \bigcap_{u \in X_{s} \backslash \mathbf{T F}} \operatorname{orient}\left(X_{s^{\prime \prime} \rightarrow u}, X_{u \rightarrow s^{\prime \prime}}\right) .
\end{aligned}
$$

We call the (10) coregulation $\left(s^{\prime}, s^{\prime \prime}, k, \mathbf{X}_{\mathbf{S}}, \theta\right)$ and its associated propagation rule is described by the following:

$$
\frac{T=\left|\left\{\left(s^{\prime}, s^{\prime \prime}, t\right) \mid \max \left(D_{s^{\prime} \rightarrow t}\right) \leq \theta \wedge \max \left(D_{s^{\prime \prime} \rightarrow t}\right) \leq \theta\right\}\right|,|\mathbf{S} \backslash \mathbf{T}|=k}{\bigwedge_{X_{i} \in \mathbf{S} \backslash \mathbf{T}} D_{i}=D_{i} \cap(\theta, 1]} .
$$

Expressing biological hypotheses and network properties as constraints may assist the phase of experimental design for GRN inference. The solver verifies the existence of a set of solutions consistent with the hypotheses, and its size can be related to confidence strength of the answer with respect to the collective prediction decisions. Consider the case where a gene is inaccurately identified as a transcription factor, or if none of its targets are identified among its putative target genes $X_{s}$. In such a case, the CSP will return no valid models. ${ }^{5}$ In order to revise the model and generate valid solutions, a relaxation of the $t f$ constraint is required. This can be achieved by either removing such constraint from the model or by changing the putative target set $X_{s}$. In the case where $X_{s}$ includes the entire set of genes in the network, and the model is still unsatisfiable, then, it can be claimed that, with respect to the knowledge leveraged by the CN prediction ensemble, such transcription factor has no direct effects on the genes analyzed, and the associated tf constraint can be removed. When performing inference on gene expression data, analysis of gene subnetworks may hide known global properties. It is crucial to test the biological value of a model prior to generating the GRN prediction.

\subsection{Solution Search}

The proposed modeling of GRN prediction allows a significant degree of flexibility in exploring the solution space. We implement an incomplete search strategy that explores the prop-labeling tree (i.e., the search space of assignments to the variablessee Section 2.1) making use of Monte Carlo (MC) methods, via a leftmost (fixed) variable selection strategy or prioritizing the variables $X_{s \rightarrow t}$, with $s \in \mathbf{T F}$, when the TF set is known. We visit the prop-labeling tree executing a random choice (i.e., a random value selection) when nondeterministic choices occur. After every nondeterministic choice is done, the propagation rules described earlier are applied to possibly reduce the nondeterminism of unlabeled variables. The search stops after a given number of trials or when a given number of solutions have been found.

\subsection{GRN Consensus}

A challenge in GRN inference is the absence of a widely accepted objective function to drive the solution search. We decided to generate an ensemble of $m$ solutions and propose three criteria to compute the final GRN prediction. Given a set of $m$ solutions $\mathbf{S}=\left\{S_{1}, \ldots, S_{m}\right\}$, where each $S_{i}=\left\langle a_{1}^{i}, \ldots, a_{n^{2}-n}^{i}\right\rangle$, let $\left.\mathbf{S}\right|_{X_{k}}=\bigcup_{i=1}^{m}\left\{a_{k}^{i}\right\}$ be the set of values

\footnotetext{
${ }^{5}$ Accordingly to the interaction patterns detected by the prediction methods employed in the committee.
} 

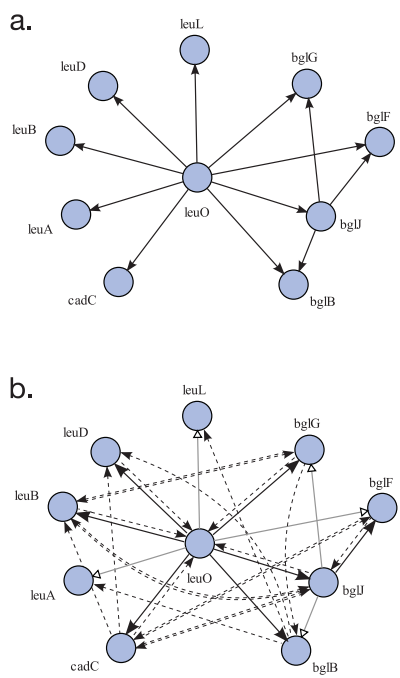
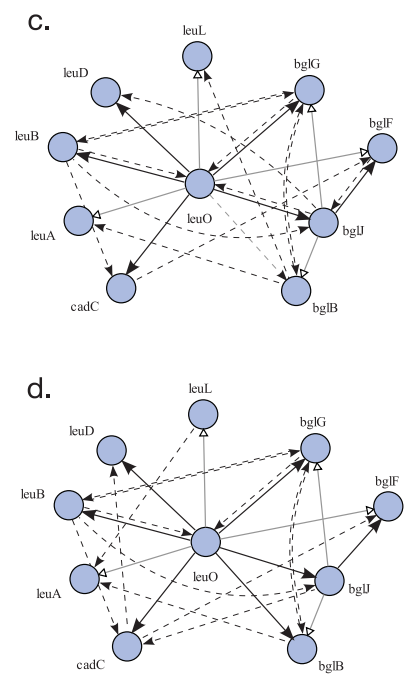
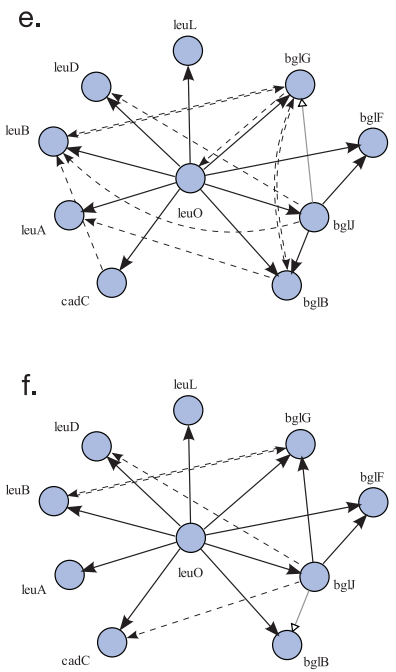

Fig. 4. An extract of 10 node E. coli GRN (a) (from Gama-Castro et al. [2008]) and its $\mathrm{CN}_{\text {rank }}$ consensus prediction (b). The CCN predictions after the integration of the sparsity constraint (c), the redundant constraint (d), the t-factor constraints (e), and co-reg (f).

assigned to the variable $X_{k}^{6}$ in the different solutions, and $\operatorname{freq}(S, a, k)$ be the function counting the occurrences of the value $a$ among the assignments to $X_{k}$ in the solution set $S$. The consensus value $a_{k}^{*}$ associated with the variable $x_{k}$ is computed by

-Mode: $a_{k}^{*}=\arg \max _{\left.a \in \mathbf{S}\right|_{X_{k}}}[\operatorname{freq}(S, a, k)]$. This estimator rewards the edge confidence value appearing with the highest frequency in the solution set. The intuition is that edge-specific confidence values appearing in many solutions may be important for the satisfaction of the constraints.

-Average: $a_{k}^{*}=\frac{1}{m} \sum_{i=1}^{m} a_{k}^{i}$. It computes the average edge consensus among all solutions in order to capture recurring predictive trends.

-Hamming distance: $a_{k}^{*}=a_{k}^{h}$, where $h=\min _{i} \sum_{j} H D\left(S_{i}, S_{j}\right), H D\left(S_{i}, S_{j}\right)=$ $\sum_{k}\left|l\left(X_{k}^{i}\right)-l\left(X_{k}^{j}\right)\right|$, and $l\left(X_{k}^{i}\right)$ is the position of the value $a_{k}^{i}$ in the domain $D_{k}$, whose elements are listed in increasing order. This measure is a global measure, that acts collectively on the prediction values of all edges, returning the solution which minimizes the Hamming distance among all edge prediction values.

\subsection{A Case Study}

We provide an example to illustrate our approach. We extract a subnetwork of 10 nodes from the $E$. coli regulatory network (Figure 4(a)) and simulate its dynamics using GeneNetWeaver (GNW) [Schaffter et al. 2011] - a standard software for GRN inference evaluation. The target network has two transcription factors (leuO and bglJ) which are in turn coregulators for genes $b g l G, b g l F, b g l B$, and it has 11 interactions.

Phase 1: CN Predictions. The prediction methods employed to construct the final CN are (i) BC3NET, (ii) CLR, (iii) GENIE3, (iv) GLN, (v) Inferelator, Pearson Correlation, and (vii) Tigress. The prediction ensemble is obtained by feeding a multifactorial expression dataset composed of 10 measurements to each of the aforementioned methods. The data is generated via GNW. In addition, we generate a CN, $\mathrm{CN}_{\text {rank }}$ (Figure 4(b)), by averaging the ranks obtained within each individual prediction, as done in Marbach

\footnotetext{
${ }^{6}$ For readability we write here $X_{k}$ rather than $X_{i \rightarrow j}$.
} 
Table II. The Prediction Accuracy of the $\mathrm{CN}_{\text {rank }}$ and CCNs on the 10-Node "E. coli" GRN

\begin{tabular}{|l|cc|cc|cc|cc|cc|}
\hline & \multicolumn{2}{|c|}{ CN $_{\text {rank }}$} & \multicolumn{2}{c|}{ CCN $_{\text {best }}$} & \multicolumn{2}{c|}{ CCN $_{\text {avg }}$} & \multicolumn{2}{c|}{ CCN $_{\text {mode }}$} & \multicolumn{2}{c|}{ CCN $_{\text {hd }}$} \\
CCNs & AUROC & AUPR & AUROC & AUPR & AUROC & AUPR & AUROC & AUPR & AUROC & AUPR \\
\hline $\mathrm{sp}$ & 0.716 & 0.302 & 0.831 & 0.4 & 0.789 & 0.328 & 0.671 & 0.283 & 0.745 & 0.291 \\
$+\mathrm{re}$ & 0.716 & 0.302 & 0.855 & 0.396 & 0.814 & 0.341 & 0.73 & 0.304 & 0.773 & 0.326 \\
$+\mathrm{tf}$ & 0.716 & 0.302 & 0.942 & 0.551 & 0.848 & 0.415 & 0.777 & 0.366 & 0.824 & 0.421 \\
$+\mathrm{cr}$ & 0.716 & 0.302 & 0.95 & 0.659 & 0.902 & 0.498 & 0.777 & 0.366 & 0.781 & 0.299 \\
\hline
\end{tabular}

et al. [2012], and use it as a baseline to build the domain variables (see Algorithm 1) and for evaluation.

Phase 2: Modeling the CSP. The execution of Algorithm 1 for the prediction disagreements analysis reduces the initial domain sizes to 1 for 21 cases, and to 3 for the others. We automatically tuned the parameters of the algorithm, as described in Section 4.3.1.

A sparsity constraint is imposed at the global level:

$$
\text { atleast_k_ge }\left(k_{l}, \mathbf{X}, \theta_{l}\right) \cap \text { atmost_k_ge }\left(k_{m}, \mathbf{X}, \theta_{m}\right) \text {, }
$$

where $\mathbf{X}$ are the variables describing all possible interactions of the network.

As the inference ensemble adopted employs methods that may suffer from the edge redundancy problem, we impose a redundant constraint for all the edge pairs $(s, t),(t, s)$ that satisfy the redundant property [see (7)] as

$$
\operatorname{red}-\mathrm{e}(t, s) \rightarrow \operatorname{orient}\left(X_{s \rightarrow t}, X_{t \rightarrow s}\right) \text {. }
$$

This constraint is able to reduce the value uncertainty for 12 additional variablesonly one element in their domains can possibly satisfy the aforementioned conditions for any value choice of the required edge variable.

Phase 3: Generating the Consensus. We perform 1,000 Monte Carlo trials producing a set of solutions which we refer to as Constrained Community Networks (CCNs).

To illustrate the effects of constraint integration on the CCNs, we consider the best prediction returned by each CSP exhibiting a different combination of the imposed constraints. We plot it as a graph containing all and only the edges of highest confidence necessary to make such graph weakly connected. These resulting predictions are illustrated in Figures 4(c) and 4(d), together with the $\mathrm{CN}_{\text {rank }}(\mathrm{b})$. In each network, the thick edges denote the true positive predictions, the dotted edges denote the false positive predictions, and the gray edges with white arrows denote the false negatives. The results are also summarized in Table II, where we report the AUROC and AUPR scores [Baldi et al. 2000] for the best prediction $\left(\mathrm{CCN}_{\text {best }}\right)$ generated and for each $\mathrm{CCN}$ generated by the evaluation criteria presented in in Section 3.4: Mode $\left(\mathrm{CCN}_{\text {mode }}\right)$, Average $\left(\mathrm{CCN}_{\mathrm{avg}}\right)$, and Hamming distance $\left(\mathrm{CCN}_{\mathrm{hd}}\right){ }^{7}$

Phase 4: Employing Network-Specific Information. Let us now model some specific information about the target network. The target network includes two TFs, leuO and bglJ, which can be modeled via two t-factor constraints as

$$
\begin{aligned}
& \text { atleast_k_ge }\left(k, \mathbf{X}_{l e u O}, \theta\right) \cap \bigcap_{u \in X_{\text {leu } O} \backslash\{l e u O, b g l J\}} \operatorname{orient}\left(X_{l e u O \rightarrow u}, X_{u \rightarrow l e u O}\right) \text {, } \\
& \text { atleast_k_ge }\left(k, \mathbf{X}_{b g l J}, \theta\right) \cap \bigcap_{u \in X_{b g l J} \backslash\{l e u O, b g l J\}} \operatorname{orient}\left(X_{b g l J \rightarrow u}, X_{u \rightarrow b g l J}\right),
\end{aligned}
$$

with $\mathbf{X}_{i}=\left\{X_{i \rightarrow j} \in \mathbf{X} \mid j \in V\right\}$ for $i=\operatorname{leu} O, \operatorname{bglK}$, and the parameters $k$ and $\theta$ set as described in Section 4.3.1. Figure 4 and Table II show the improvements using this

\footnotetext{
${ }^{7}$ AUROC and AUPR are popular measures from the machine learning literature—see Section 4.2.
} 
final formalization. Finally, speculation about the activity of genes leuO and $b g l J$ as coregulators can be captured via a co-reg constraint expressed by

$$
\text { coregulation }\left(l e u O, \text { bglJ, } k, \mathbf{X}_{\mathbf{S}}, \theta\right) \cap \bigcap_{u \in X_{s} \backslash\{l e u O, b g l J\}} \operatorname{orient}\left(X_{l e u O \rightarrow u}, X_{u \rightarrow l e u O}\right),
$$

where $\mathbf{X}_{S}=\left\{X_{s \rightarrow t} \mid s \in\{l e u O, \operatorname{bgl} J\}, t \in V\right\}$ is the set of all candidate regulations having $l e u O$ or bgle as TF, and $k$ and $\theta$ set as described in Section 4.3.1. The application of this additional constraint produces further improvements (Figure 4 and Table II).

\section{PERFORMANCE EVALUATION}

We systematically assessed the ability of the CCN schema to accurately reconstruct GRNs both in an ab initio scenario (only datasets information available) and in the presence of prior information in biologically relevant settings. We started by conducting experiments to select a subset of methods to be used in the committee schema to construct a CN. We assess the power of each constraint individually and how the constraint interaction affects the performance predictions. Finally, we evaluate the performance of the CCNs predictions against the CN schema. Performance is validated against the set of gold standard GRNs.

\subsection{Benchmark Networks and Datasets}

The benchmark networks adopted to asses the performance of our method were produced extracting subnetworks from the E. coli [Alon 2007; Gama-Castro et al. 2008] and Saccharomyces cerevisiae [Guelzim et al. 2002; Reguly et al. 2006] regulatory networks. The datasets used to simulate the dynamics of such networks were generated in GeneNetWeaver [Schaffter et al. 2011], a tool commonly adopted for the generation of synthetic GRN benchmarks, and used to generate the synthetic datasets for the DREAM3 [Prill et al. 2010] and DREAM4 [Greenfield et al. 2010] competitions.

We adopt two types of steady-state expression data: ${ }^{8}$

(1) Multifactorial: These are measurements obtained by (slightly) perturbing all genes simultaneously. Multifactorial data might correspond, for example, to expression profiles obtained from different patients or biological replicates. Such type of data is simpler and less expensive to obtain than other types of data, such as knockout/knockdown or time series data, and is thus more common in practice; however, it is also less informative for the prediction of edge directionality [Bansal et al. 2007] and therefore makes the regulatory network inference task more challenging.

(2) Knockout: These are steady-state levels of a single-gene knockout (deletion). The datasets are built by performing an independent knockout for a subset of genes. A knockout experiment is simulated by setting the gene's transcription rate to zero.

The complete benchmark network set adopted in this work is composed of 20 large GRNs, each described by three datasets: one containing multifactorial data, one containing knockout data, and another one containing both data types. We will refer to these three datasets as $m f, k o$, and $a l l$, respectively. The benchmark set is composed of

(1) FDP Networks: five networks of sizes 100 extracted from the $E$. coli and another five networks of the same size from the $S$. cerevisiae regulatory networks, denoted respectively by $\mathrm{E}_{i}$ and $\mathrm{S}_{i}$ with $i=1, \ldots, 5$. The associated datasets are generated via GNW, by setting the value of Seed to random and the Neighbor selection to $20 \%$. The model was generated by producing 100 microarray datasets, enabling both the ODE

\footnotetext{
${ }^{8}$ Experiments where the mRNA expressions are observed once when at a steady state.
} 

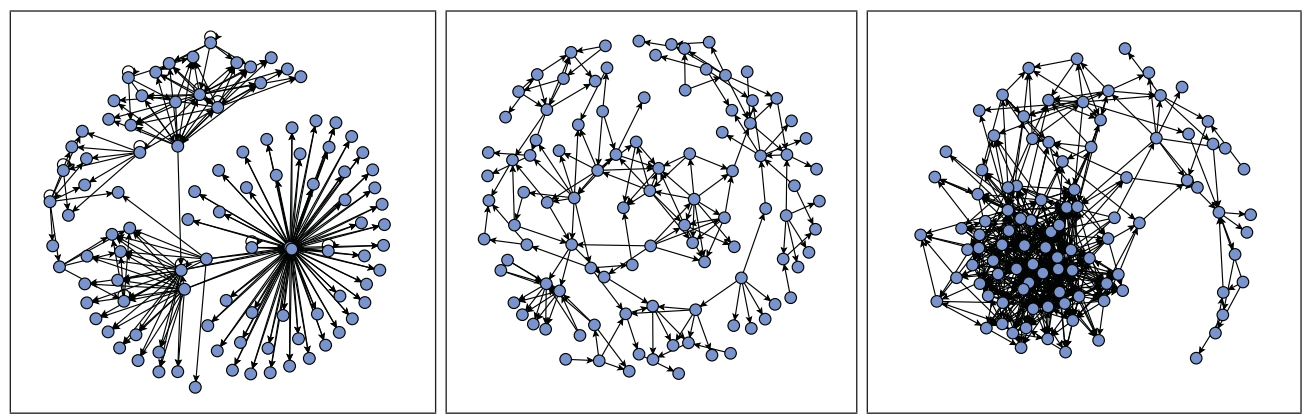

Fig. 5. Gene regulatory networks with IDs E 2 (left), D3 3 (center), and D35 (right).

Table III. Properties of the Benchmark Network Topologies. (*) Gama-Castro et al. [2008],

$\left(^{* \star}\right)$ Balaji et al. [2006], $\left(^{\star \star \star}\right)$ Shen-Orr et al. [2002], and $\left(^{\star \star \star \star}\right)$ Reguly et al. [2006].

\begin{tabular}{|l|lccc||c|lccc|}
\hline ID & \multicolumn{1}{|c}{ GRN } & Edges & TFs & Hubs & ID & GRN & Edges & TFs & Hubs \\
\hline $\mathrm{E}_{1}$ & E. coli $(*)$ & $148(4)$ & 17 & 8 & $\mathrm{~S}_{1}$ & S. cerevisiae $(* *)$ & $174(0)$ & 17 & 9 \\
$\mathrm{E}_{2}$ & E. coli $(*)$ & $151(6)$ & 19 & 7 & $\mathrm{~S}_{2}$ & S. cerevisiae $(* *)$ & $205(0)$ & 18 & 9 \\
$\mathrm{E}_{3}$ & E. coli $(*)$ & $218(6)$ & 18 & 7 & $\mathrm{~S}_{3}$ & S. cerevisiae $(* *)$ & $207(0)$ & 18 & 6 \\
$\mathrm{E}_{4}$ & E.coli $(*)$ & $159(4)$ & 17 & 7 & $\mathrm{~S}_{4}$ & S. cerevisiae $(* *)$ & $168(0)$ & 18 & 9 \\
$\mathrm{E}_{5}$ & E. coli $(*)$ & $171(10)$ & 20 & 5 & $\mathrm{~S}_{5}$ & S. cerevisiae $(* *)$ & $202(0)$ & 20 & 7 \\
\hline \hline $\mathrm{D}_{1}$ & $E . \operatorname{coli}(* * *)$ & $125(0)$ & 26 & 11 & $\mathrm{D}_{1}$ & Synthetic $(* * *)$ & $176(14)$ & 40 & 8 \\
$\mathrm{D}_{2}$ & $E . \operatorname{coli}(* * *)$ & $119(0)$ & 19 & 7 & $\mathrm{D}_{2}$ & Synthetic $(* * *)$ & $249(14)$ & 35 & 7 \\
$\mathrm{D}_{3}$ & Yeast $(* * *)$ & $166(0)$ & 59 & 26 & $\mathrm{D}_{3}$ & Synthetic $(* * *)$ & $195(6)$ & 44 & 17 \\
$\mathrm{D}_{4}$ & Yeast $(* * * *)$ & $389(0)$ & 70 & 25 & $\mathrm{D}_{4}$ & Synthetic $(* * *)$ & $211(8)$ & 40 & 14 \\
$\mathrm{D}_{5}$ & Yeast $(* * * *)$ & $551(0)$ & 80 & 30 & $\mathrm{D}_{5}$ & Synthetic $(* * *)$ & $193(4)$ & 34 & 15 \\
\hline
\end{tabular}

and the Stochastic Differential Equation (SDE) options, with the SDE value equal to 0.05 . The noise level was set to simulate the microarray standard noise with default parameters.

(2) D3: five networks from the DREAM3 competition [Prill et al. 2010], denoted by $\mathrm{D}_{1}, \ldots, \mathrm{D} 3_{5}$ and consisting of 100 genes, built by extracting modules from the $E$. coli and from a yeast genetic interaction network [Reguly et al. 2006]. Data normalization was made by the competition organizers.

(3) D4: five GRNs from the DREAM4 competition [Greenfield et al. 2010], denoted by D $4_{1}, \ldots, \mathrm{D}_{5}$ and consisting of 100 genes. The network topologies have been extracted from the transcriptional regulatory networks of $E$. coli and $S$. cerevisiae. The data corresponds to noisy measurements of mRNA levels based on SDEs (Langevin equations) and has been normalized to values in $[0,1]$.

The GRNs adopted in this study are representative of a diverse range of heterogeneous network topologies, varying properties such as sparsity, number of hubs, and local connectivity-as illustrated in Figure 5. A summary of the network topologies used in the performance assessment is given in Table III, where we report the network ID, the transcription regulatory network from where the network has been extracted, the total number of gene-gene interactions (edges) and the number of bidirectional regulatory interactions (in parentheses), the number of TFs, and the number of network hubs-defined as the number of nodes whose out-degree exceeds the average TFs out-degree for the same GRN.

We adopt the datasets associated with the Fioretto-Dovier-Pontelli (FDP) GRNs to assess the individual methods prediction performance and to train the construction of the $\mathrm{CN}$ prediction under the Borda rank [Borda 1971] election schema $\left(\mathrm{CN}_{\text {rank }}\right)$. This 
results in 4320 individual predictions and $360 \mathrm{CN}$ predictions. We use the complete collection of datasets to assess the performances of the CCNs against the $\mathrm{CN}$ predictions.

\subsection{Performance Assessment}

To measure prediction accuracy we evaluate each prediction as a binary classification task-where interactions are predicted as either being present or absent. The ranked list of interactions is compared against a gold standard via two measures largely adopted in machine learning: the area under the precision versus recall curve (AUPR) and the area under the receiver operating characteristic curve (AUROC)-true positive rate versus false-positive rate [Baldi et al. 2000]. To compute the AUROC and the AUPR curves, we express the measures of True Positive Rate (TPR), False-Positive Rate (FPR), precision, and recall as functions of a cutoff threshold $(k)$, which denotes the number of ranked edges to be considered. Let us denote with $P$ the number of interactions in the gold standard, with $N$ the number of negatives (absent interactions), and with $T=P+N$ the total number of putative edges.

The true-/false-positive rates and precision and recall are defined as follows:

$$
T P R(k)=\frac{T P(k)}{P}, \quad F P R(k)=\frac{F P(k)}{N}, \quad \operatorname{precision}(k)=\frac{T P(k)}{T P(k)+F P(k)}, \quad \operatorname{recall}(k)=\frac{T P(k)}{P},
$$

where $T P(k)[F P(k)]$ is the number of true correct (incorrect) predictions among the first $k$ elements in the interaction list. An AUROC value of 0.5 (1.0) corresponds to a random (perfect) prediction. AUPR values close to 0 indicate the predominance of erroneous predictions, while a value of 1.0 denotes a prediction with no errors.

\subsection{Settings}

We performed the various experiments using the $R$ language and a generic CSP solver, capable of handling real valued variables and of exploring the search space using a prop-labeling tree with random value selection (Section 3.4). We use $R$ to generate the GRN predictions for each method presented in Section 2.2 and to assess their individual performance and the performance as committees in ranked-based CNs. The parameters associated with each prediction method have been set to the default values, and the predictions generated are fed to the constraint solver. The constraint solver generates the constrained CNs. Our CSP solver explores the queue of constraints using techniques based on the notion of event (a change in the domain of a variable) [Schulte and Stuckey 2008] and is implemented in C++.

For each experiment, we perform 10,000 Monte Carlo trials and generate the CCNs using all of the solutions found. We generate four CCN consensus solutions, one for each estimator described in Section $3.4\left(\mathrm{CCN}_{\text {mode }}, \mathrm{CCN}_{\mathrm{avg}}, \mathrm{CCN}_{\mathrm{hd}}\right)$ and $\mathrm{CCN}_{\text {best }}$, as the best prediction with respect to the AUROC score. We notice that the CCN produced via the mode and avg estimators may outperform the $\mathrm{CCN}_{\text {best }}$, as they generate a new solution, starting from those found during the search phase, which may not be part of such ensemble. All the experiments have been performed on an Intel Core i7 3770 machine, $3.4 \mathrm{GHz}$ with $16 \mathrm{~GB}$ of RAM, equipped with the SuSE Linux operative system.

4.3.1. Automatic Parameter Tuning. Let us now discuss the algorithms and constraints parameters tuning adopted by default by the system. In what follows, we will assume that the edge predictions in $\mathrm{CN}_{\text {rank }}$ are sorted from the most likely one to the least likely one, and we will refer to $\mathrm{CN}_{\text {rank }}[i]$ as the confidence value assigned to the highest

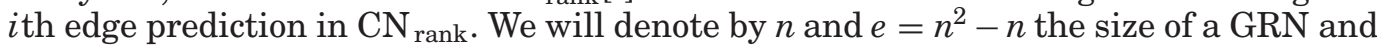
the number of putative regulators, respectively.

Domain construction: The domains analysis and reduction phase, described in $\mathrm{Al}$ gorithm 1 is used to assess the community prediction disagreements on a given edge. Recall that the bounds $L$ and $U$ are used to discriminate whether the confidence for the 
presence/absence of an interaction is strong within the CN prediction. Their values are selected to be, respectively, the highest confidence value in the last $\mathrm{CN}_{\text {rank }}$ decile and the lowest confidence value in the first $\mathrm{CN}_{\text {rank }}$ decile. The disagreement threshold $\theta_{d}$ is set to the average of the discrepancy values w_d across all the edges of the network. A summary of the parameters values is reported as follows:

$$
L=\mathrm{CN}_{\text {rank }}[0.9 e], \quad U=\mathrm{CN}_{\text {rank }}[n], \quad \theta_{d}=\frac{1}{|E|} \sum_{(s, t) \in E}\left(\frac{1}{\left(\begin{array}{l}
J \\
2
\end{array}\right)} \sum_{j=1}^{J} \sum_{i=j+1}^{J}\left|\omega_{j}^{\#}(s, t)-\omega_{i}^{\#}(s, t)\right|\right) .
$$

We also tried to populate domains in a nonsymmetric way, for example, by looking at how many methods lie inside which quantile and by assigning a value representative of each quantile. This method led to worst results with respect to the one adopted in our experiments.

Sparsity constraint: To guide the parameter selection for the sparsity constraint, we set the values $k_{l}$ and $k_{m}$ (see Equation (4)) to be, respectively, $n$ and $n \log (n)$, since the number of edges in a sparse network is considered to be $O(n \log (n))$.

To identify the thresholds $\theta_{l}$ and $\theta_{m}$, we select for each gene the minimum (maximum, respectively) weight $\bar{w}$, such that the number of outgoing edges in the $\mathrm{CN}_{\text {rank }}$ predicted with confidence greater than $\bar{w}$ is greater than (less than or equal to, respectively) $1(\log (n)$, respectively) and average these values. By doing so, we try to impose a restrictive condition for the satisfaction of the atleast_k_ge and atmost_k_ge constraints. The sparsity constraint values are summarized next:

$$
\begin{aligned}
& k_{l}=n, \quad \theta_{l}=\frac{1}{n} \sum_{s \in V} \theta_{l}^{s}, \quad k_{m}=n \log (n), \quad \theta_{m}=\frac{1}{n} \sum_{s \in V} \theta_{m}^{s}, \\
& \theta_{l}^{s}=\min _{\bar{w}}\left|\left\{(s, t, w) \in \mathrm{CN}_{\text {rank }} \mid t \in V, w \geq \bar{w}\right\}\right|>1, \\
& \theta_{m}^{s}=\max _{\bar{w}}\left|\left\{(s, t, w) \in \mathrm{CN}_{\text {rank }} \mid t \in V, w \geq \bar{w}\right\}\right| \leq \log (n) .
\end{aligned}
$$

Redundant edge constraint: The parameters $\beta_{i}$ introduced in Equation (7) for the redundant edge definition are set to the mean of all the differences of the confidence values of the pairs of edges $(s, t)$ and $(t, s)$ in each $\mathrm{CN}$ ensemble prediction $P_{i} \in \mathcal{P} \backslash \mathcal{H}$ (see Equation (7)):

$$
\beta_{i}=\frac{1}{|E|} \sum_{(s, t) \in E}\left(w_{s \rightarrow t}^{i}-w_{t \rightarrow s}^{i}\right)
$$

where $w_{s \rightarrow t}^{i}$ is the confidence value associated with the edge $(s, t)$ reported by $P_{i}$.

We also tried to learn the values of the $\beta_{i}$ of Equation (7) using a set of 10 training networks. In our study we compare the confidence values assigned to the true edges $w_{s \rightarrow t}$ - present in the gold standard-against the confidence assigned to the opposite edges $w_{t \rightarrow s}$ - true negatives-for each individual prediction $P_{i}$ in $\mathcal{P} \backslash \mathcal{H}$ and estimate the $\beta_{i} \geq 0$ solving the following linear program:

$$
\begin{aligned}
\text { maximize } & \left|\left\{\left(w_{s \rightarrow t}^{i}-w_{t \rightarrow s}^{i}\right)>\beta_{i} \mid(s, t) \in E_{G S} \wedge(t, s) \notin E_{G S}\right\}\right|, \\
\text { subjected to } & \left|\left\{\left(w_{s \rightarrow t}^{i}-w_{t \rightarrow s}^{i}\right)>\beta_{i} \mid(s, t) \notin E_{G S} \wedge(t, s) \in E_{G S}\right\}\right|=0,
\end{aligned}
$$

where $E_{G S}$ is the set of edges in the gold standard. Applying the determined $\beta_{i}$ to Equation (7) in our experiments resulted in almost all cases in detecting no redundant edges.

Transcription factor constraint: The atleast_k_ge constraint parameters $k$ and $\theta$ employed to express a t-factor constraint are automatically set so that $k=\log (n)$ and $\theta=\mathrm{CN}_{\text {rank }}[k]$. To guarantee the constraint satisfaction, we add the value $\theta$ in the domains of those variables $X_{i}$ involved in the tf constraint, having $\max \left(D_{i}\right)<\theta$. 
Table IV. Ranked Method Lists for the Multifactorial Dataset (left), the Knockout

Dataset (center), and the Combined Dataset (right)

\begin{tabular}{|r|lcc|lcc|lcc|}
\hline$\#$ & met & AUROC & AUPR & met & AUROC & AUPR & met & AUROC & AUPR \\
\hline 1 & $\underline{\text { tigr }}$ & 0.710 & 0.141 & $\underline{\text { tigr }}$ & 0.797 & 0.109 & $\underline{\text { tigr }}$ & 0.789 & 0.164 \\
2 & $\underline{\text { geni }}$ & 0.725 & 0.120 & $\frac{\text { geni }}{\text { cr }}$ & 0.770 & 0.070 & $\underline{\text { geni }}$ & 0.802 & 0.143 \\
3 & $\underline{\text { lr }}$ & 0.690 & 0.107 & $\underline{\text { pear }}$ & 0.770 & 0.069 & $\underline{\text { pear }}$ & 0.754 & 0.089 \\
4 & $\underline{\text { pear }}$ & 0.694 & 0.097 & $\underline{\text { gln }}$ & 0.693 & 0.057 & $\underline{\text { infe }}$ & 0.707 & 0.103 \\
5 & mrne & 0.688 & 0.102 & $\underline{\text { bc3n }}$ & 0.627 & 0.063 & $\underline{\text { bc3n }}$ & 0.653 & 0.112 \\
6 & kend & 0.684 & 0.097 & $\underline{\text { infe }}$ & 0.672 & 0.045 & $\underline{\text { gln }}$ & 0.667 & 0.072 \\
7 & $\underline{\text { bc3n }}$ & 0.636 & 0.112 & $\underline{\text { clr }}$ & 0.557 & 0.024 & $\underline{\text { clr }}$ & 0.659 & 0.077 \\
8 & spea & 0.683 & 0.094 & spea & 0.553 & 0.024 & spea & 0.657 & 0.067 \\
9 & $\underline{\text { infe }}$ & 0.639 & 0.091 & kend & 0.553 & 0.024 & kend & 0.657 & 0.067 \\
10 & $\underline{\text { gln }}$ & 0.653 & 0.082 & mrne & 0.553 & 0.024 & mrne & 0.655 & 0.067 \\
11 & arac & 0.597 & 0.086 & arac & 0.532 & 0.026 & arac & 0.574 & 0.058 \\
12 & c3ne & 0.589 & 0.085 & c3ne & 0.520 & 0.027 & c3ne & 0.564 & 0.056 \\
\hline
\end{tabular}

Coregulator constraint: The values for the co-reg constraint parameters $k$ and $\theta$ (see Equation (7)) are the same as those used in the tf constraint. Also in this case, we expand the domain of the variables involved as described in the previous paragraph.

To guarantee the satisfaction of the constraints orient $\left(X_{s \rightarrow t}, X_{t \rightarrow s}\right)$, we introduce a value 0 in the domains of the variables $X_{t \rightarrow s}$ if $\min \left(D_{t \rightarrow s}\right) \geq \max \left(D_{s \rightarrow t}\right)$.

\subsection{Analysis of Individual Methods and Community Network Construction}

To construct the CN schema, we evaluate each individual prediction over the complete collection of datasets associated with the FDP networks. For each dataset $(m f, k o$, and $a l l$ ) we assess the performance of the prediction methods from Section 2.2, by averaging the AUROC and AUPR values associated with each prediction of the FDP benchmark networks. In Table IV, we report, for each dataset, the list of the methods sorted according to the quality of their performance. The final CN schema results in a selection of 7 of the 12 GRN prediction methods. We consider both the methods GENIE3 and Tigress because of their different approaches in performing the feature selection step: GENIE3 relies on random forests while Tigress uses a regression step. We also discriminate the method BC3NET from the other MI-based models, as it employs an additional bagging step. For each data type ( $m f, k o$, and $a l l)$, the final methods ensemble selected to form the CN includes Tigress, GENIE3, Pearson Correlation, GLN, BC3Net, Inferelator, and CLR - these appear underlined in Table IV. All other methods fall in a category already represented in the ensemble. A report of the performance of the individual methods is provided in the Appendix, in Tables I- IV.

\subsection{Analysis of Individual Constraint in the $\mathrm{CCN}$}

In this section, we analyze the construction of the CCNs by measuring the impact of the application of individual constraints on the quality of the resulting solution ensemble. The tests are performed over the datasets associated with the FDP networks and, for each experiment, we report the median predictions found with respect to the AUROC score. Figure 6 illustrates the impact of each constraint on the AUROC score for each network of the FDP multifactorial dataset. The plot reports the median of a set of 10,000 solutions associated with the CCNs generated exploiting only the redundant constraint ( $r e$ ), the sparsity constraint ( $\mathrm{sp}$ ), the t-factor constraint ( $\mathrm{t} f$ ), and the co-reg constraint (cr), together with the trace of all the solutions generated via an unconstrained problem with same settings (gray stripe). The results for the datasets based on $k o$ and all data follow the same trend. 


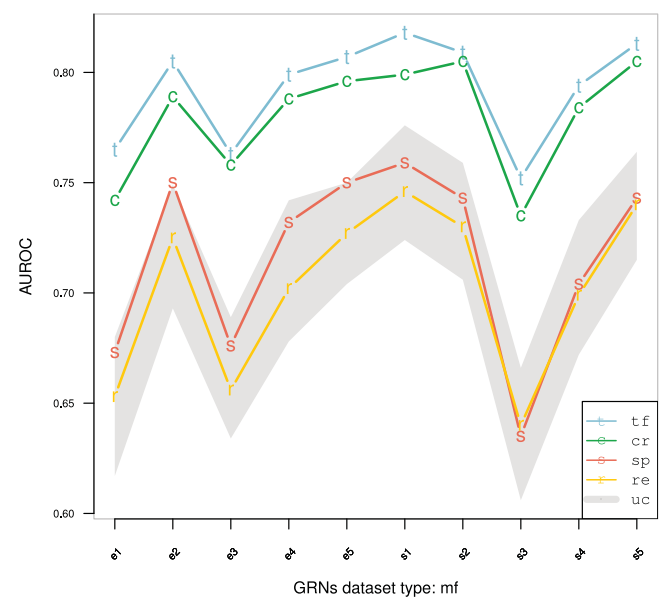

Fig. 6. The impact of each constraint on the AUROC score for the multifactorial dataset in the FDP networks.
Table V. Summary of the Type of Edges Detected by the redundant Constraint

\begin{tabular}{|cc|cccc|}
\hline Net & Data & $\mathrm{tf} \rightarrow \mathrm{tg}$ & $\mathrm{tf} \rightarrow \mathrm{tf}$ & $\mathrm{tg} \rightarrow \mathrm{tf}$ & $\mathrm{tg} \rightarrow \mathrm{tg}$ \\
\hline $\mathrm{E}_{1}$ & $m f$ & 4 & 0 & 0 & 6 \\
$\mathrm{E}_{2}$ & $m f$ & 4 & 0 & 0 & 16 \\
$\mathrm{E}_{3}$ & $m f$ & 0 & 1 & 0 & 6 \\
$\mathrm{E}_{4}$ & $m f$ & 5 & 1 & 1 & 10 \\
$\mathrm{E}_{5}$ & $m f$ & 3 & 0 & 0 & 11 \\
$\mathrm{~S}_{1}$ & $m f$ & 4 & 0 & 1 & 13 \\
$\mathrm{~S}_{2}$ & $m f$ & 6 & 0 & 1 & 11 \\
$\mathrm{~S}_{3}$ & $m f$ & 6 & 0 & 0 & 20 \\
$\mathrm{~S}_{4}$ & $m f$ & 7 & 0 & 1 & 17 \\
$\mathrm{~S}_{5}$ & $m f$ & 4 & 0 & 0 & 13 \\
\hline \multicolumn{5}{|c|c}{ Average } \\
\hline FDP & $m f$ & 4.30 & 0.2 & 0.4 & 12.30 \\
FDP & $k o$ & 2.5 & 0.1 & 0.90 & 16.60 \\
FDP & $a l l$ & 7.1 & 0.2 & 1.20 & 17.4 \\
\hline
\end{tabular}

We observe that the median values of the solutions generated using the sparsity constraint are close to the best solutions generated in the unconstrained problem; this confirms the effectiveness of the constraint. The biological information encoded by the $t$-factor and co-reg constraints enhances the quality of the solutions beyond the capabilities of the unconstrained search. As for the redundant constraint, the median solutions returned are slightly better that the median solutions found by the unconstrained problem. To validate the effectiveness of such constraint, we examine the number of redundant edges correctly identified via the red-e property $(\mathrm{t} f \rightarrow \mathrm{tg}$ and $\mathrm{tg} \rightarrow \mathrm{tg}$ )—where tf denotes a TF gene and tg a gene targeted by some TF, but not itself a TF-and the number of edges wrongly predicted as redundant $(\mathrm{t} f \rightarrow \mathrm{tf}$ and $\mathrm{tg} \rightarrow \mathrm{tf}$ ). In Table V, we report the extended results for the multifactorial dataset (top) and we summarize the results for all three datasets in the bottom part of the table. Observe that the constraint is more effective in the $m f$ dataset, where fewer errors occur.

Let us analyze the impact of combining two constraints. We adopt the same settings as in the previous experiments. The results are reported in Figure 7. The plots illustrate the median of a set of 10,000 solutions associated with the CCNs generated via a combination of the sparsity constraint (top left), the redundant constraint (top right), the $t$-factor constraint (bottom left), and the co-reg constraint (bottom right) with all the others. As for Figure 6, we mark with a gray stripe the scores for the solutions generated by the unconstrained CCNs. The knockout and the combined datasets follow the same trend as those in Figure 7. Observe that the interaction among constraints is important to improve the quality of the solutions. An evaluation of the impact of each constraint combination on the FDP networks is reported in the Appendix, Figures I(a) $\mathrm{I}(\mathrm{m})$.

\subsection{Constrained Community Networks versus Community Networks}

To assess the ability of the CCN approach to accurately reconstruct GRNs, we focus on two subproblems: (1) We examine the predicted CCNs using only general network topological information, such as the sparsity and the redundant constraints, to leverage community-method features and networks properties; (2) We integrate network-specific biological knowledge available. Due to the observations made in the previous section, 

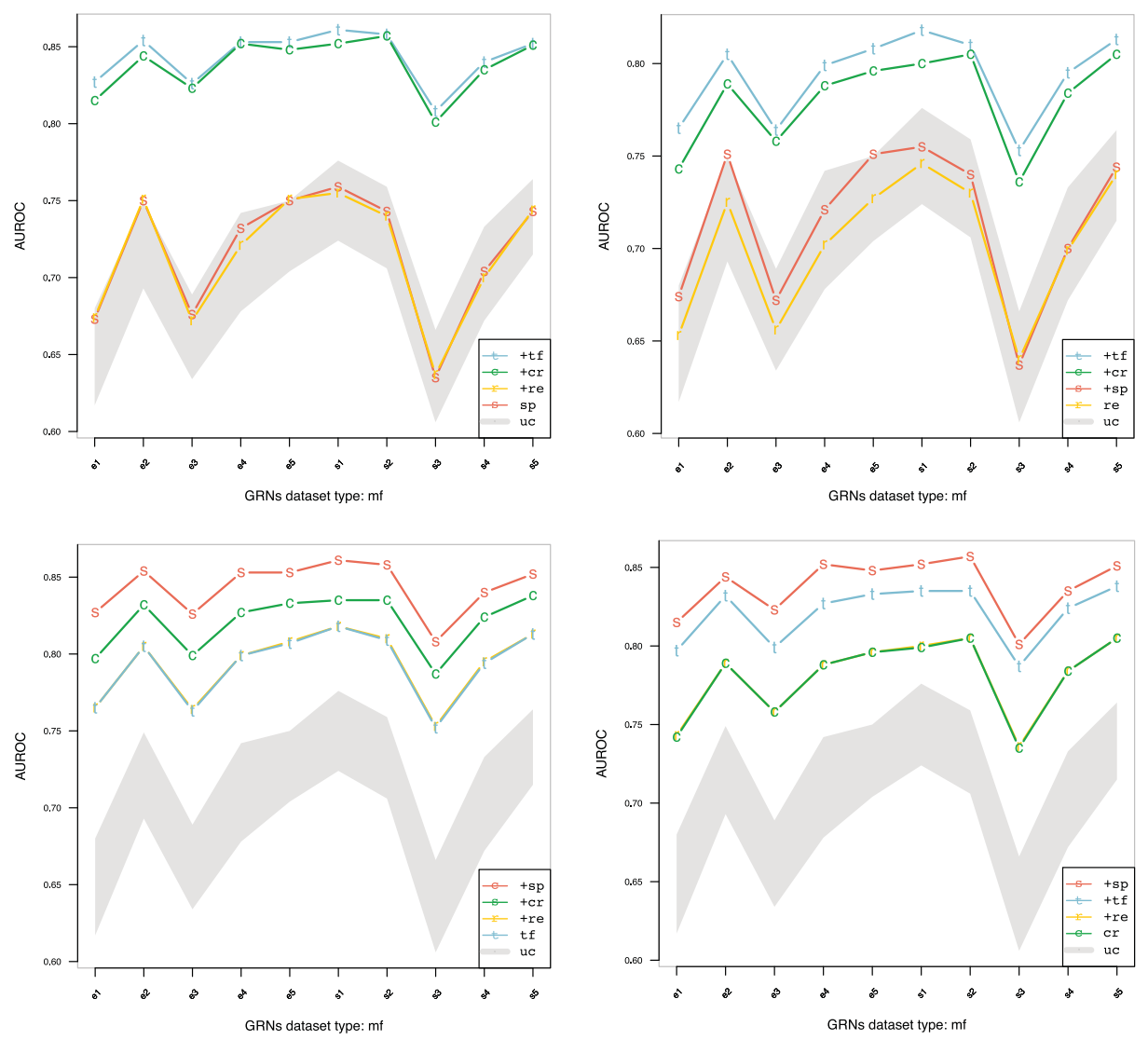

Fig. 7. The impact on the AUROC scores for the multifactorial dataset in the FDP networks when combining two constraints fixing the sparsity (top left), the redundant (top right), the t-factor (bottom left), or the co-reg (bottom right) constraints.

Table VI. AUROC and AUPR \% Improvements for the CCN with best, Average (avg), Hamming Distance (hd), and mode Estimators with Respect to the $\mathrm{CN}_{\text {rank }}$ in Multifactorial Data

\begin{tabular}{|c|c|c|c|c|c|c|c|c|c|c|c|c|}
\hline \multirow[b]{2}{*}{ AUROC } & \multicolumn{4}{|c|}{$F D P$} & \multicolumn{4}{|c|}{ DREAM3 } & \multicolumn{4}{|c|}{ DREAM4 } \\
\hline & best & avg & hd & mode & best & avg & hd & mode & best & avg & hd & mode \\
\hline $\mathrm{sp}(+\mathrm{re})$ & 1.163 & 1.083 & 1.033 & 0.963 & 1.108 & 1.028 & 1.028 & 1.108 & 0.859 & 0.839 & 0.839 & 0.839 \\
\hline$+\mathrm{tf}$ & 17.543 & 18.883 & 13.623 & 5.483 & 18.948 & 18.108 & 15.308 & 9.648 & 24.939 & 24.799 & 21.539 & 12.919 \\
\hline$+\mathrm{cr}$ & 18.333 & 21.223 & 16.203 & 5.483 & 20.888 & 22.148 & 17.768 & 9.648 & 27.459 & 29.479 & 24.559 & 12.919 \\
\hline \multicolumn{13}{|l|}{ AUPR } \\
\hline $\mathrm{sp}(+\mathrm{re})$ & 1.173 & 0.434 & 0.273 & 0.189 & 0.296 & 0.046 & 0.040 & 0.050 & 0.053 & 0.029 & 0.025 & 0.027 \\
\hline$+\mathrm{tf}$ & 9.488 & 8.568 & 6.291 & 4.658 & 2.716 & 1.542 & 1.570 & 0.732 & 3.307 & 1.699 & 2.089 & 0.627 \\
\hline$+\mathrm{cr}$ & 5.658 & 12.238 & 1.878 & 4.658 & 4.936 & 2.448 & 2.866 & 0.732 & 5.715 & 2.585 & 3.969 & 0.627 \\
\hline
\end{tabular}

we opt not to involve the redundant constraint for the datasets containing knockouts data. We categorize the benchmarks by datasets ( $m f, k o$, and $a l l)$ and networks (FDP, D3, and D4), and average their respective AUROC and AUPR scores. Tables VI, VII, and VIII report the average AUROC and AUPR improvements (as a percentage) for the networks inferred from the $m f, k o$, and all datasets, respectively, of the $\mathrm{CCN}_{\text {best }}$, $\mathrm{CCN}_{\mathrm{avg}}, \mathrm{CCN}_{\mathrm{hd}}$, and $\mathrm{CCN}_{\text {mode }}$ with respect to $\mathrm{CN}_{\text {rank }}$. We first focus on the results of 
Table VII. AUROC and AUPR \% Improvements for the CCN with best, Average (avg), Hamming Distance (hd), and mode Estimators with Respect to the $\mathrm{CN}_{\text {rank }}$ in the Knockout Data

\begin{tabular}{|c|c|c|c|c|c|c|c|c|c|c|c|c|}
\hline \multirow[b]{2}{*}{ AUROC } & \multicolumn{4}{|c|}{$F D P$} & \multicolumn{4}{|c|}{ DREAM3 } & \multicolumn{4}{|c|}{ DREAM4 } \\
\hline & best & avg & hd & mode & best & avg & hd & mode & best & avg & hd & mode \\
\hline sp $(+r e)$ & 0.529 & 0.409 & 0.369 & 0.319 & -0.517 & -0.577 & -0.777 & -0.677 & 0.261 & 0.201 & 0.161 & 0.021 \\
\hline$+t f$ & 11.159 & 13.459 & 8.669 & 3.719 & 1.843 & 6.763 & -0.577 & 3.123 & 6.801 & 11.021 & 4.181 & 5.401 \\
\hline$+\mathrm{cr}$ & 11.889 & 15.179 & 9.819 & 3.719 & 2.223 & 8.023 & 0.783 & 3.123 & 7.201 & 12.721 & 5.341 & 5.401 \\
\hline \multicolumn{13}{|l|}{ AUPR } \\
\hline $\mathrm{sp}(+\mathrm{re})$ & 0.981 & 0.129 & 0.187 & -0.077 & 2.000 & 0.120 & -0.516 & -0.740 & 1.004 & 0.140 & 0.374 & -0.202 \\
\hline$+t f$ & 4.990 & 5.715 & 2.858 & 1.208 & 2.940 & 5.140 & -0.220 & 2.520 & 3.130 & 5.790 & 0.414 & 2.970 \\
\hline$+\mathrm{cr}$ & 7.248 & 9.038 & 3.822 & 1.208 & -1.180 & 8.320 & -4.016 & 2.520 & 1.300 & 8.530 & -1.416 & 2.970 \\
\hline
\end{tabular}

Table VIII. AUROC and AUPR \% Improvements for the CCN with best, Average (avg), Hamming Distance (hd), and mode Estimators with Respect to the $\mathrm{CN}_{\text {rank }}$ in the Combined (all) Data

\begin{tabular}{|c|c|c|c|c|c|c|c|c|c|c|c|c|}
\hline \multirow[b]{2}{*}{ AUROC } & \multicolumn{4}{|c|}{$F D P$} & \multicolumn{4}{|c|}{ DREAM3 } & \multicolumn{4}{|c|}{ DREAM4 } \\
\hline & best & avg & hd & mode & best & avg & hd & mode & best & avg & hd & mode \\
\hline sp (+re) & 1.266 & 1.136 & 1.106 & 1.026 & -0.599 & -0.639 & -0.779 & -0.779 & 0.707 & 0.527 & 0.507 & 0.467 \\
\hline$+t f$ & 13.106 & 14.936 & 10.206 & 4.526 & 2.061 & 6.361 & -0.479 & 2.941 & 22.947 & 21.987 & 19.607 & 11.967 \\
\hline$+\mathrm{cr}$ & 13.796 & 16.636 & 11.796 & 4.526 & 2.361 & 7.801 & 0.761 & 2.941 & 24.087 & 24.907 & 20.347 & 11.967 \\
\hline \multicolumn{13}{|l|}{ AUPR } \\
\hline sp (+re) & 1.282 & 0.400 & 0.464 & 0.161 & 2.178 & 0.118 & 0.338 & -0.802 & 0.251 & 0.075 & 0.033 & 0.047 \\
\hline$+\mathrm{tf}$ & 8.394 & 8.454 & 4.827 & 4.008 & 3.338 & 4.598 & -0.902 & 2.038 & 3.433 & 2.303 & 2.051 & 1.057 \\
\hline$+\mathrm{cr}$ & 5.064 & 12.514 & 1.794 & 4.008 & -0.614 & 7.458 & -2.880 & 2.038 & 5.961 & 3.303 & 3.131 & 1.057 \\
\hline
\end{tabular}

the CCNs obtained when only the sparsity (sp) and the redundant (re) constraints are active, given the aforementioned restrictions (first row of each table).

For the $m f$ datasets (Table VI), the CCNs outperform the CN schema in every benchmark network. For the datasets that include knockout data ( $k o$ and all in Tables VII and VIII, respectively), the CCNs increase the prediction accuracy with respect to the $\mathrm{CNs}$ for the FDP and the $D 4$ networks; the only exception is the mode estimator, which produces a slight performance degradation in the $k o$ dataset. The AUROC measures for the $D 3$ networks result in a performance degradation ranging from $0.52 \%$ to $0.78 \%$. On the other hand, the precision versus recall score produces an enhancement of the prediction accuracy up to $2.8 \%$ for the best estimator.

As shown in Fioretto and Pontelli [2013], the prediction accuracy for the CCNs is consistent and often better than that of a CN schema in the ab initio scenario.

It is interesting to observe that the CCNs consistently outperform the CNs prediction in the multifactorial datasets. This result is appealing, as such type of data has been shown to be less informative than knockout or time-series data, while it is substantially cheaper to produce and more abundant.

The next experiment extends the set of constraints adopted to model the GRNs to include specific knowledge about individual networks. We enable the t-factor ( $t f$ ) and the co-reg constraints ( $\mathrm{cr}$ ) over the set of genes which are known to be TFs or coregulators in the target networks. The results are reported in Tables VI, VII, and VIII in the second and third rows for the addition of the tf and cr constraints, respectively. The integration of such additional knowledge results in significant improvements of the GRN predictions, both in terms of AUROC (up to 29.5\%) and AUPR scores (up to 15.2\%). This result supports our hypothesis that the addition of biological knowledge can better guide the predictions, even when the same inference ensemble is used.

Let us also observe that the best improvements in terms of AUROC and AUPR scores can be found in the CCN with the average estimator, and this is true for all the networks considered and every dataset, including the ones with knockout data. 


\section{CONCLUSIONS}

In this article, we introduced the CCNs paradigm to solve the gene regulatory network inference problem. CCNs use constraint programming techniques to guide the integration of predictions in a CN.

The use of constraints to model topological and biologically relevant prior information of a regulatory network provides several advantages in the creation of CNs. Our approach does not impose any hypothesis on the datasets adopted nor on the type of inference methods. Furthermore, constraints can naturally handle heterogeneous knowledge, facilitating the balancing of the strengths and weaknesses of the individual inference methods composing the inference ensemble.

We introduced a class of constraints able to (1) guarantee GRNs' specific properties and (2) take into account the community prediction collective agreements on each edge, and the limitations of each specific method. Experiments performed over a set of more than 300 benchmarks, including large networks proposed in the DREAM challenges, show that our approach can consistently outperform the consensus networks constructed by averaging individual edges ranks, as proposed in Marbach et al. [2012].

We have shown how the integration of knowledge about target networks acquired in biological relevant settings can provide significant improvements in terms of GRN prediction quality when compared to a state-of-the-art CN approach (up to $29.5 \%$ and $15.2 \%$ for AUROC and AUPR measures, respectively). This was possible as our model encourages the modular integration of biological knowledge, in the form of logical rules.

As part of our future work, we plan to investigate new optimization measures that take into account local and global network properties, for example, the number of specific network motifs in a target GRN region or the scale-free degree in a given portion of the graph. This can be achieved by including soft constraints in our model. We plan to use this information to address method-specific biases toward different connectivity patterns. On the CP side, we will extend existing constraints, for instance, by studying the most likely set where a t-factor constraint could be targeted, and model new constraints and propagators to capture different types of biological knowledge, such us information about cell line and conditions at the time of the experiment, or the information encoded in functional modules-groups of TFs which regulate a particular biological process. Moreover we plan to employ path-based constraints, for example, acting on the cascade effects resulting in a reward or penalty of an edge confidence value.

\section{ELECTRONIC APPENDIX}

The electronic appendix for this article can be accessed in the ACM Digital Library.

\section{ACKNOWLEDGMENTS}

We thank the anonymous reviewers for their comments.

\section{REFERENCES}

D. Allocco, I. Kohane, and A. Butte. 2004. Quantifying the relationship between co-expression, co-regulation and gene function. BMC Bioinf. 5, 1 (2004), 18+.

U. Alon. 2007. Network motifs: Theory and experimental approaches. Nat. Rev. Genet. 8, 6 (2007), 450-461.

G. Altay, M. Asim, F. Markowetz, and D. E. Neal. 2011. Differential C3NET reveals disease networks of direct physical interactions. BMC Bioinf. 12 (2011), 296.

G. Altay and F. E. Streib. 2010. Inferring the conservative causal core of gene regulatory networks. BMC Syst. Biol. 4, 1 (2010), 132+.

K. Apt. 2009. Principles of Constraint Programming. Cambridge University Press. 
S. Balaji, M. M. Babu, L. M. Iyer, N. M. Luscombe, and L. Aravind. 2006. Comprehensive analysis of combinatorial regulation using the transcriptional regulatory network of yeast. J. Mol. Biol. 360, 1 (2006), 213-227.

P. Baldi, S. Brunak, Y. Chauvin, C. A. Andersen, and H. Nielsen. 2000. Assessing the accuracy of prediction algorithms for classification: An overview. Bioinformatics 16, 5 (2000), 412-424.

M. Bansal, V. Belcastro, A. Ambesi-Impiombato, and D. Di Bernardo. 2007. How to infer gene networks from expression profiles. Mol. Syst. Biol. 3 (2007), 78.

A. Bauer-Mehren, L. I. Furlong, and F. Sanz. 2009. Pathway databases and tools for their exploitation: Benefits, current limitations and challenges. Mol. Syst. Biol. 5, 1 (2009).

C. M. Bishop and N. M. Nasrabadi. 2006. Pattern Recognition and Mmachine Learning. Vol. 1. Springer.

R. Bonneau et al. 2006. The inferelator: An algorithm for learning parsimonious regulatory networks from systems-biology data sets de novo. Genome Biol. 7, 5 (2006), R36.

J. C. Borda. 1971. Memoire sur les elections au scrutin.

L. Bortolussi and A. Policriti. 2008. Modeling biological systems in stochastic concurrent constraint programming. Constraints 13, 1-2 (2008).

L. Breiman, J. Friedman, C. J. Stone, and R. A. Olshen. 1984. Classification and Regression Trees. Chapman $\&$ Hall, New York, NY.

F. Corblin, E. Fanchon, and L. Trilling. 2010. Applications of a formal approach to decipher discrete genetic networks. BMC Bioinf. 11 (2010), 385.

F. Corblin, S. Tripodi, E. Fanchon, D. Ropers, and L. Trilling. 2009. A declarative constraint-based method for analyzing discrete genetic regulatory networks. Biosystems 98, 2 (2009), 91-104.

H. De Jong. 2002. Modeling and simulation of genetic regulatory systems: A literature review. J. Comput. Biol. 9, 1 (2002), 67-103.

R. de Matos Simoes and F. Emmert-Streib. 2012. Bagging statistical network inference from large-scale gene expression data. PloS ONE 7, 3 (2012), e33624+.

P. D'Haeseleer, X. Wen, S. Fuhrman, and R. Somogyi. 1999. Linear modeling of mRNA expression levels during CNS development and injury. In Pacific Symposium on Biocomputing. 41-52.

F. Eduati, J. De Las Rivas, G. Di Camillo, G. Toffolo, and J. Saez-Rodriguez. 2012. Integrating literatureconstrained and data-driven inference of signalling networks. Bioinformatics 28, 18 (2012), 2311-2317.

B. Efron, T. Hastie, I. Johnstone, and R. Tibshirani. 2004. Least angle regression. Ann. Statist 32, 2 (2004), 407-499.

F. Fages, G. Batt, E. D. Maria, D. Jovanovska, A. Rizk, and S. Soliman. 2010. Computational systems biology in BIOCHAM. ERCIM News 2010, 82, 36.

J. J. Faith, B. Hayete, J. T. Thaden, I. Mogno, J. Wierzbowski, G. Cottarel, S. Kasif, J. J. Collins, and T. S. Gardner. 2007. Large-scale mapping and validation of escherichia coli transcriptional regulation from a compendium of expression profiles. PLoS Biol. 5, 1 (2007), e8.

F. Fioretto and E. Pontelli. 2013. Constraint programming in community-based gene regulatory network inference. In CMSB 2013 (LNBI), Vol. 8130. Springer-Verlag, 135-149.

S. Gama-Castro, V. Jiménez-Jacinto, M. Peralta-Gil, A. Santos-Zavaleta, M .I. Peñaloza-Spinola, B. Contreras-Moreira, J. Segura-Salazar, L. Muñiz-Rascado, I. Martínez-Flores, and H. Salgado. 2008. RegulonDB: Gene regulation model of Escherichia coli K-12 beyond transcription, active annotated promoters and Textpresso navigation. Nucleic Acids Res. 36, D120-D124.

M. Gebser, T. Schaub, S. Thiele, and P. Veber. 2008. Detecting inconsistencies in large biological networks with answer set programming. In Logic Programming, LNCS, Vol. 5366. Springer, Berlin, 130-144.

A. Greenfield, A. Madar, H. Ostrer, and R. Bonneau. 2010. DREAM4: Combining genetic and dynamic information to identify biological networks and dynamical models. PLoS ONE 5, 10 (2010), e13397.

N. Guelzim, S. Bottani, P. Bourgine, and F. Képès. 2002. Topological and causal structure of the yeast transcriptional regulatory network. Nat. Genet. 31, 1 (April 2002), 60-63.

M. A. Harris and Gene Ontology Consortium. 2004. The Gene Ontology (GO) database and informatics resource. Nucleic Acids Res. 32, Database issue (2004), D258-D261.

T. Hastie, R. Tibshirani, and J. Friedman. 2009. The Elements of Statistical Learning: Data Mining, Inference and Prediction (2nd. ed.). Springer, New York, NY.

S. Kim, S. Imoto, and S. Miyano. 2003. Dynamic Bayesian network and nonparametric regression for nonlinear modeling of gene networks from time series gene expression data. Biosystems 104-113.

S. K. Kummerfeld and S. A. Teichmann. 2006. DBD: A transcription factor prediction database. Nucleic Acids Res. 34, Suppl. 1 (2006), D74-D81. 
L. P. Lim, N. C. Lau, P. Garrett-Engele, A. Grimson, J. M. Schelter, J. Castle, D. P. Bartel, P. S. Linsley, and J. M. Johnson. 2005. Microarray analysis shows that some microRNAs downregulate large numbers of target mRNAs. Nature 433, 7027 (2005), 769-773.

P. B. Madhamshettiwar, S. R. Maetschke, M. J. Davis, A. Reverter, and M. A. Ragan. 2012. Gene regulatory network inference: Evaluation and application to ovarian cancer allows the prioritization of drug targets. Genome Med. 4, 5 (1 May 2012), 41+.

D. Marbach, J. C. Costello, R. Küffner, N. M. Vega, R. J. Prill, D. M. Camacho, and the DREAM5 Consortium. 2012. Wisdom of crowds for robust gene network inference. Nat. Meth. 9, 8 (2012), 796-804.

A. A. Margolin, I. Nemenman, K. Basso, C. Wiggins, G. Stolovitzky, R. D. Favera, and A. Califano. 2006. ARACNE: An algorithm for the reconstruction of gene regulatory networks in a mammalian cellular context. BMC Bioinf. 7, Suppl. 1 (2006).

N. Meinshausen and P. Bühlmann. 2010. Stability selection. J. R. Stat. Soc.: Ser. B (Statistical Methodology) 72, 4 (Sept. 2010), 417-473.

P. E. Meyer, K. Kontos, F. Lafitte, and G. Bontempi. 2007. Information-theoretic inference of large transcriptional regulatory networks. EURASIP J. Bioinf. Sys. Biol. (2007). DOI : 10.1155/2007/79879

M. P. Perrone. 1993. Improving Regression Estimation: Averaging Methods for Variance rReduction with Extensions to General Convex Measure Optimization. Ph.D. dissertation. Brown University, Providence, RI.

R. J. Prill, D. Marbach, J. Saez-Rodriguez, P. K. Sorger, L. G. Alexopoulos, X. Xue, N. D. Clarke, G. AltanBonnet, and G. Stolovitzky. 2010. Towards a rigorous assessment of systems biology models: The DREAM3 challenges. PLoS ONE 5, 2 (2010), e9202.

T. Reguly, A. Breitkreutz, L. Boucher, B. Breitkreutz, G. C. Hon, C. L. Myers, A. Parsons, H. Friesen, R. Oughtred, and A. Tong. 2006. Comprehensive curation and analysis of global interaction networks in Saccharomyces cerevisiae. J. Biol. 5, 4 (08 June 2006), 11+.

M. Renda and U. Straccia. 2003. Web metasearch: Rank vs. score based rank aggregation methods. In Proceedings of the 2003 ACM Symposium on Applied Computing. ACM, New York, NY, 841-846.

T. Schaffter, D. Marbach, and D. Floreano. 2011. GeneNetWeaver: In silico benchmark generation and performance profiling of network inference methods. Bioinformatics 27, 16 (June 2011), 2263-2270.

C. Schulte and P. J. Stuckey. 2008. Efficient constraint propagation engines. ACM Trans. Prog. Lang. Syst. $31,1$.

S. S. Shen-Orr, R. Milo, S. Mangan, and U. Alon. 2002. Network motifs in the transcriptional regulation network of Escherichia coli. Nat. Genet. 31, 1 (2002), 1061-4036.

A. Sîrbu, H. J. Ruskin, and M. Crane. 2012. Integrating heterogeneous gene expression data for gene regulatory network modelling. Theor. Biosci. 131, 2 (2012), 95-102.

T. Soh and K. Inoue. 2010. Identifying necessary reactions in metabolic pathways by minimal model generation. In ECAI 2010. IOS Press, Amsterdam, The Netherlands, 277-282.

M. J. Song, C. K. Lewis, E. R. Lance, E. J. Chesler, R. K. Yordanova, M. A. Langston, K. H. Lodowski, and S. E. Bergeson. 2009. Reconstructing generalized logical networks of transcriptional regulation in mouse brain from temporal gene expression data. EURASIP J. Bioinf. Syst. Biol. (2009), 5.

N. Sun and H. Zhao. 2009. Reconstructing transcriptional regulatory networks through genomics data. Stat. Meth. Med. Res. 18, 6 (2009), 595-617.

R. Thomas. 1973. Boolean formalization of genetic control circuits. J. Theor. Biol. 42, 3, 563-585.

S. Videla, C. Guziolowski, F. Eduati, S. Thiele, N. Grabe, J. Saez-Rodriguez, and A. Siegel. 2012. Revisiting the training of logic models of protein signaling networks with ASP. In Comput. Meth. Syst. Biol. LNCS, Vol. 7605, 342-361.

X. Zhou, X. Wang, and E. Dougherty. 2006. Genomic Networks: Statistical Inference from Microarray Data. John Wiley \& Sons.

Received January 2014; revised August 2014; accepted October 2014 